\title{
Black hole formation in core-collapse supernovae and time-of-flight measurements of the neutrino masses
}

\author{
J. F. Beacom* \\ Physics Department 161-33, California Institute of Technology, Pasadena, California 91125 \\ and NASA/Fermilab Astrophysics Center, Fermi National Accelerator Laboratory, Batavia, Illinois 60510-0500 \\ R. N. Boyd ${ }^{\dagger}$ \\ Departments of Astronomy and Physics, The Ohio State University, Columbus, Ohio 43210
}

A. Mezzacappa

Physics Division, Oak Ridge National Laboratory, Oak Ridge, Tennessee 37831

(Received 19 October 2000; published 7 March 2001)

\begin{abstract}
In large stars that have exhausted their nuclear fuel, the stellar core collapses to a hot and dense protoneutron star that cools by the radiation of neutrinos and antineutrinos of all flavors. Depending on its final mass, this may become either a neutron star or a black hole. Black hole formation may be triggered by mass accretion or a change in the high-density equation of state. We consider the possibility that black hole formation happens when the flux of neutrinos is still measurably high. If this occurs, then the neutrino signal from the supernova will be terminated abruptly (the transition takes $\lesssim 0.5 \mathrm{~ms}$ ). The properties and duration of the signal before the cutoff are important measures of both the physics and astrophysics of the cooling protoneutron star. For the event rates expected in present and proposed detectors, the cutoff will generally appear sharp, thus allowing model-independent time-of-flight mass tests for the neutrinos after the cutoff. If black hole formation occurs relatively early, within a few $(\sim 1)$ seconds after core collapse, then the expected luminosities are of order $L_{B H}=10^{52} \mathrm{erg} / \mathrm{s}$ per flavor. In this case, the neutrino mass sensitivity can be extraordinary. For a supernova at a distance $D=10 \mathrm{kpc}$, SuperKamiokande can detect a $\bar{\nu}_{e}$ mass down to $1.8 \mathrm{eV}$ by comparing the arrival times of the high-energy and low-energy neutrinos in $\bar{\nu}_{e}+p \rightarrow e^{+}+n$. This test will also measure the cutoff time, and will thus allow a mass test of $\nu_{\mu}$ and $\nu_{\tau}$ relative to $\bar{\nu}_{e}$. Assuming that $\nu_{\mu}$ and $\nu_{\tau}$ are nearly degenerate, as suggested by the atmospheric neutrino results, masses down to about $6 \mathrm{eV}$ can be probed with a proposed lead detector of mass $M_{D}=4$ kton (OMNIS). Remarkably, the neutrino mass sensitivity scales as $\left(D / L_{B H} M_{D}\right)^{1 / 2}$. Therefore, direct sensitivity to all three neutrino masses in the interesting few-eV range is realistically possible; there are no other known techniques that have this capability.
\end{abstract}

DOI: 10.1103/PhysRevD.63.073011

PACS number(s): 14.60.Pq, 04.70.-s, 97.60.Bw, 97.60.Lf

\section{INTRODUCTION}

In the past several years, the growing evidence for neutrino oscillations has caused a great deal of excitement over the implied nonzero neutrino masses. Oscillation phenomena, however, are sensitive only to differences of the squared neutrino masses, and thus provide only a lower bound on the heavier mass. Without further input, the deduced masses can be increased, and the difference of masses decreased, providing exactly the same difference of squared masses and hence the same oscillation phenomena.

It is therefore of crucial importance to experimentally measure or constrain the absolute scale of the neutrino masses. Two indirect techniques have been proposed. First, the sum of the neutrino masses can be constrained by cosmological arguments. The requirement of not overclosing the universe gives an upper bound of about $100 \mathrm{eV}$ [1]. This bound may be improved by considering the effects of neu-

\footnotetext{
*Electronic address: beacom@fnal.gov

†Electronic address: boyd@mps.ohio-state.edu

‡Electronic address: mezz@nova.phy.ornl.gov
}

trino masses on the cosmic microwave background and the clustering of galaxies; the claimed (future, in some cases) sensitivity is about $1-10 \mathrm{eV}$ [2]. These arguments require that the other cosmological parameters are independently known and may not apply if the neutrinos decay. ${ }^{1}$ Second [4], if all of the neutrino masses are connected by small measured mass-squared differences, then each mass is constrained by the limit on the electron neutrino mass from tritium beta decay, now about $3 \mathrm{eV}$ [5] (the direct laboratory limits on the mu and tau neutrino masses are $170 \mathrm{keV}$ [6] and $18 \mathrm{MeV}$ [7], respectively). If neutrinoless double beta decay were discovered (i.e., neutrinos were confirmed to have a Majorana character), then this could anchor the masses at an even lower value [8]; the present limit on the combination of masses measured in double beta decay is about $0.2 \mathrm{eV}$ [9]. Strictly speaking, to use the arguments of

\footnotetext{
${ }^{1}$ Furthermore, it has recently been shown that in scenarios with a low (MeV-scale) reheating temperature, the neutrinos may decouple without reaching equilibrium, leading to a substantially lower density than in the usual scenario; this may weaken the cosmological neutrino mass bounds by a factor of 10 or more [3]
} 
Refs. [4,8], each oscillation signal must first be decisively confirmed, including precise measurement of the mixing parameters and identification of the oscillated flavors. Until then, we must allow for the possibility that there are more relevant flavors than there are measured mass-squared differences. For example, if the solar neutrino problem is solved by $\nu_{e} \rightarrow \nu_{s}$ oscillations, and if the Liquid Scintillation Neutrino Detector (LSND) signal is ruled out, then the atmospheric neutrino problem can be solved by $\nu_{\mu} \rightarrow \nu_{\tau}$ oscillations with a small mass difference and large masses, say 10 or $100 \mathrm{eV}$, as long as $\delta m^{2} \simeq 10^{-3} \mathrm{eV}^{2}[10]$.

Thus, while the indirect constraints on the neutrino masses are valuable, it would be much more satisfying to have a direct experimental measurement. Presently, the best possibility for direct measurement of the mu and tau neutrino masses is by time-of-flight differences using neutrinos from a Galactic core-collapse supernova. ${ }^{2}$ At lowest order, a neutrino with mass $m$ (in $\mathrm{eV}$ ) and energy $E$ (in $\mathrm{MeV}$ ) will experience an energy-dependent delay (in s) relative to a massless neutrino in traveling over a distance $D$ (in $10 \mathrm{kpc}$ ):

$$
\Delta t(E)=0.515\left(\frac{m}{E}\right)^{2} D
$$

The distance is scaled by the approximate distance to the Galactic center, though a supernova may be detected from anywhere in the Galaxy and its immediate companions (e.g., the Magellanic Clouds). SuperKamiokande (SK) and the Sudbury Neutrino Observatory (SNO) would have good sensitivity to a Galactic supernova, collecting of order $10^{4}$ and $10^{3}$ events, respectively (see Refs. [12-14] and references therein). Unless the decreasing neutrino luminosity is interrupted by black hole formation, it should be possible to measure it to very late times (some tens of seconds); either outcome would be an important probe of the nuclear equation of state $[15,16]$.

The primary interest for mass tests is to measure the mu and tau neutrino masses relative to the nearly massless electron neutrino. A neutrino mass test $[12,13]$ based on the average event arrival times $\langle t\rangle$ can measure a mu or tau neutrino mass as small as $45 \mathrm{eV}$ in $\mathrm{SK}$ and $30 \mathrm{eV}$ in SNO. If the mu and tau neutrinos are maximally mixed with nearly degenerate masses, then the sensitivity on either mass eigenstate is better by a factor of about $\sqrt{2}$, i.e., about $30 \mathrm{eV}$ in SK and $20 \mathrm{eV}$ in SNO [12,13]. This test is independent of supernova neutrino emission models, though it does assume that the luminosities of the different flavors have similar

\footnotetext{
${ }^{2}$ As noted by Shrock [11], if neutrinos are mixed, then beta decay spectra consist of incoherent contributions from each mass eigenstate, where the endpoints depend on the masses, and the weighting on the mixing angles. The presence of kinks in the spectrum would thus allow direct measurement of $m_{2}$ and $m_{3}$ and their mixing angles. In order to experimentally separate such kinks from an endpoint turnover due to $m_{1}$, the mass differences and the mixing angles must be large enough. For light neutrinos, the $\bar{\nu}_{e}$ disappearance experiments presently provide more restrictive limits on these parameters.
}

shapes as a function of time, as expected on general grounds and also seen in the supernova models [17]. In the absence of a model, a Kolmogorov-Smirnov test can be used to compare the event rates for different flavors of neutrinos; it can be shown that this reduces to the $\langle t\rangle$ test [18]. Other tests proposed in the literature are explicitly model-dependent, and the models have large uncertainties.

While the $\langle t\rangle$ test could improve the limit on the tau neutrino mass by almost six orders of magnitude, it seems very difficult to reach the $\mathrm{eV}$ range suggested by the cosmological and tritium arguments above. It can be shown [13] that the mass sensitivity generically scales with the detector mass $M_{D}$ as $1 / M_{D}^{1 / 4}$; therefore, another order of magnitude in sensitivity in neutrino mass would require detectors $10^{4}$ times larger, which seems impossible. It can also be shown [13] that the sensitivity is independent of the distance to the supernova in the case where the deduced neutrino mass is compatible with zero and only an upper limit is placed.

In this paper, a comprehensive study that follows our recent Letter [19], we consider the case that the proto-neutron star forms a black hole, instead of gradually cooling as a stable neutron star. If that happens early enough, then the neutrino signals will be abruptly terminated as the neutrinospheres are enveloped by the event horizon of the black hole. In Sec. II, we discuss the conditions required for this to happen and to be observable, as well as the expected details of the neutrino signal. In Sec. III, we derive the mass effects on the detected neutrino event rate in the general case. In Sec. IV, we show how to measure the black hole cutoff time in SuperKamiokande, with or without the complicating effects of a possible electron neutrino mass. In Sec. V, we show how to make a time-of-flight mass measurement of the mu and tau neutrino masses relative to the cutoff time measured in SK. Finally, in Sec. VI, we discuss some remaining issues and conclude.

\section{BLACK HOLE FORMATION AND THE SUPERNOVA NEUTRINO SIGNAL}

Before discussing how to measure the neutrino masses, we first examine how likely it is that black hole formation will truncate the neutrino flux from a Galactic supernova. Three questions naturally arise:

(1) Is the Galactic supernova rate reasonably high?

(2) Are black holes formed reasonably often in corecollapse supernovae?

(3) Can black hole formation occur when the neutrino fluxes are still high?

An examination of the evidence reveals that, while the uncertainties are large, there is a good chance of satisfying all three requirements. If so, this could have a profound impact on our ability to directly measure all three neutrino masses. Before showing how that could be done, we address these requirements.

\section{A. Galactic supernova rate}

From studies of other galaxies, we know that about 80 $90 \%$ of supernovae are of the core-collapse type (types II, 
Ib, Ic), which produce a substantial flux of neutrinos [20,21]. In the following we treat the overall supernova rate without regard to correction for the smaller rate of Ia supernovae.

A rough estimate of the Galactic supernova rate can be made using the historical records. Over the past 1000 years, 7 Galactic supernovae are known either from historical records or their remnants [21,22]. Probably some others in the southern sky were missed because they were not visible to or not recorded by the astronomers of the time. For example, the recently-discovered supernova remnant reported in Ref. [22] is apparently extremely close $(0.2 \mathrm{kpc})$ and only about 700 years old, but is not found in the historical record. It is therefore not unreasonable to estimate that nearby supernovae occur at a rate of about $1 /$ century. Due to obscuration by dust, naked-eye supernovae are not visible beyond several kpc (the farthest of these 7 was at $4.2 \mathrm{kpc}$ ); therefore, one must correct for the small fraction of the Galaxy surveyed. The Bahcall-Soneira Galactic model [23,24] includes somewhat less than $10 \%$ of the stars within about $4 \mathrm{kpc}$ of Earth; therefore, we estimate the total Galactic supernova rate to be about 10/century (see also Refs. [25,26]).

This estimate of 10/century agrees with the rate given by Bahcall and Piran [24], who make a direct integration over the stellar initial mass function, corresponding stellar lifetimes, and spatial distribution of stars; their calculation is not normalized to the historical rate. It also agrees with the nucleosynthesis arguments of Arnett, Schramm, and Truran [27].

On the other hand, more conservative estimates suggest that the rate is lower: $(3 \pm 1) /$ century [20,21]. It is not clear how to reconcile this with the above estimates of 10/century. The estimate based on the historical rate and the independent Bahcall-Piran calculation agree, and the only element they have in common is the fraction of stars nearby. Thus, the most likely fault with these calculations, if any, is that they assume that the stars that explode as supernovae are distributed in the same way as other stars. In fact, Refs. [28,29] argue against this assumption, and claim that the nearby supernova rate is anomalously high due to our occupying a privileged position in the Galaxy.

With coverage over most of the Galaxy over most of the past 20 years, no neutrino detectors have reported a Galactic supernova [30] (note that SN 1987A is excluded because it occurred in the Large Magellanic Cloud). Taken at face value, this would exclude a Galactic supernova rate of 10/ century at about the $85 \%$ C.L. However, an analysis combining all of the experiments has not been done, and is needed. A number of these experiments did not have full coverage of the Galaxy and/or had significant $(\simeq 50 \%)$ downtime, and taking this into account will yield a weaker constraint.

LIGO [31] and other novel techniques [32-34] may also be able to shed some new light on the supernova rate.

The combined evidence thus suggests a Galactic supernova rate of at least $3 /$ century.

\section{B. Relative frequency of black hole formation}

From a theoretical point of view, the relative frequency of black hole (BH) and neutron star (NS) formation (the BH/NS ratio) depends on the equation of state of nuclear matter [35] and the supernova mechanism [17]; further work on each is greatly needed. Ideally, appropriate direct observational constraints on neutron-star properties could be decisive for discriminating between different equations of state $[35,36]$.

As is well known, SN 1987A in the Large Magellanic Cloud ( $D \simeq 50 \mathrm{kpc}$ ) was clearly observed by the Kamiokande II and IMB detectors, with 12 and 8 events, respectively $[37,38]$. The observed duration of SN1987A was about $10 \mathrm{~s}$, consistent with a supernova that formed a neutron star. No neutron star has been seen yet in the remnant, but this may not mean that one is not present [39]. Thus if a black hole formed, it evidently happened after the neutrino flux died out [40]. The progenitor mass plays an important role in deciding the ultimate mass and hence fate of the core. Thus, even though SN1987A (progenitor mass $\sim 18 M_{\odot}$ ) did not form a black hole in the first $10 \mathrm{~s}$ after collapse, other supernovae will be different.

Core-collapse supernovae occur only for stars massive enough to burn their cores up to iron; this minimum mass is estimated to be about $8 M_{\odot}$. It is also generally believed that stars above some mass, perhaps $20 M_{\odot}$, will always produce black holes instead of neutron stars. Bahcall and Piran [24] estimate that supernovae from progenitors above $20 M_{\odot}$ number about $1 / 2$ of those below $20 M_{\odot}$. Ratnatunga and van den Bergh [41], with a supernova rate several times smaller, estimate about $1 / 4$ for this ratio. Fryer [42] estimates a $\mathrm{BH} / \mathrm{NS}$ ratio somewhere between a few percent and $1 / 4$, depending on the cutoff progenitor mass; both are strongly affected by the uncertainties in the inputs to his supernova code.

For an assumed stellar initial mass function, predictions of the remnant mass distribution have been made by Timmes, Woosley, and Weaver [43], who find a bimodal distribution with peaks at about $1.3 M_{\odot}$ and $1.8 M_{\odot}$. (In some other models, this bimodal distribution is not seen; see, e.g., Fig. 3 of Ref. [44]). The bimodal nature in this model is due to progenitor masses below and above $19 M_{\odot}$, which either burn carbon convectively or radiatively, respectively [43]. If the maximum neutron star mass is the conventional $2.2 M_{\odot}[45]$, then the $\mathrm{BH} / \mathrm{NS}$ ratio $\simeq 0$ [43]. However, Brown and Bethe $[46,47]$ argue that the maximum neutron star mass is about $1.5 M_{\odot}$, on the basis of both an assumed softer equation of state and a number of observational constraints. In the Brown and Bethe model, progenitors above about $18 M_{\odot}$ will form black holes, and they independently deduce a $\mathrm{BH} / \mathrm{NS}$ ratio $\sim 1$. For a maximum neutron star mass of $1.5 M_{\odot}$, the Timmes, Woosley, and Weaver remnant distribution indicates a $\mathrm{BH} / \mathrm{NS}$ ratio $\simeq 3$ (the upper peak is larger than the lower peak) [43].

Recent results by Ergma and van den Heuvel [48] indicate that the vast majority of progenitors above $20-25 M_{\odot}$ produce black holes (this therefore supports a much higher $\mathrm{BH} / \mathrm{NS}$ ratio than the earlier paper of van den Heuvel and Habets [49] that suggested a BH/NS ratio $\simeq 1 / 100$ ). This is corroborated by Ref. [50], which suggests that the progenitor mass cutoff may be even lower. These results thus suggest a high $\mathrm{BH} / \mathrm{NS}$ ratio. 
It may eventually be possible to address the absolute $\mathrm{BH} / \mathrm{NS}$ ratio observationally via the $\mathrm{BH} / \mathrm{NS}$ ratio (perhaps as large as 10 in a preliminary study [51]) deduced from lowmass x-ray binaries, though this also depends on the details of the binary evolution.

Qian, Vogel, and Wasserburg [52] assumed that the $r$-process production of heavy nuclei occurs in core-collapse supernovae and considered the effects of black hole versus neutron star formation on the yields. They found that the observed $r$-process distribution may be best explained with a very high $\mathrm{BH} / \mathrm{NS}$ ratio $\sim 10$. Their results require that black hole formation happens early, when the neutrino fluxes are relatively high, which will terminate part of the $r$-process production. While their BH/NS ratio is very large, their hypothesis is supported by recent measurements [53]. Further measurements of $r$-process yields in ultra-metal-poor stars would be very valuable.

The accumulated evidence thus supports a relatively high BH/NS ratio, so that the next Galactic supernova would be likely to form a black hole.

\section{Scenarios for black hole formation}

One scenario for black hole formation in core-collapse supernovae occurs if the proto-neutron star mass exceeds the maximum neutron star mass. For ordinary neutron-rich nuclear matter, this maximum mass is thought to be about $2.2 M_{\odot}[45]$, though there may be significant uncertainties. This may occur in the initial collapse, or after some delay, due to accretion of further mass. The neutrino signal expected in a scenario of this type has been studied by Burrows [54] and Mezzacappa and Bruenn [55] (see also a very early paper by Wilson [56]). In these models, neutrino emission was followed until abruptly terminated by black hole formation (the results do not continue through the short but nonzero black hole formation time). Before the cutoffs at 1-2 $\mathrm{s}$, the luminosities were fairly constant at more than $10^{52} \mathrm{erg} / \mathrm{s}$ per flavor.

A second scenario for black hole formation is based on a softening of the equation of state in the proto-neutron star as the neutrinos are emitted and a phase transition to a more exotic state of matter occurs, containing perhaps strange mesons or baryons, charged-pion condensates, or free quarks. The maximum neutron star mass for such exotic nuclear matter is generally lower $[46,47,57]$, perhaps about $1.5 M_{\odot}$. Thus an initially stable proto-neutron star may form a black hole after the phase transition. The details of the neutrino signal accompanying black hole formation in such scenarios have been studied by Baumgarte et al. [58]; see also earlier work [59-61]. A detailed study in full general relativity was made of the neutrino emission just before and during the formation of the black hole. A singularity-avoiding code [62] was used that tracked the emission in the frame of a distant observer (i.e., the result is the redshifted, time-dilated luminosity that would be seen in a neutrino detector). Before the cutoff at about $10 \mathrm{~s}$, the luminosities were fairly constant at about $10^{51} \mathrm{erg} / \mathrm{s}$ per flavor.

Finally, we discuss two scenarios in which a neutron star can become a black hole long after the neutrino flux has died away. As such, these scenarios are not of direct interest to us.

In a successful supernova explosion, the outgoing shock will pass through the stellar envelope within a few hours after core collapse. If a reverse shock forms, it may dump matter onto the neutron star and cause it to exceed the maximum mass, hence causing black hole formation. These fallback scenarios are discussed in Refs. [39,63].

Gradual accretion onto old neutron stars until the maximum mass is exceeded is also possible, and a concrete scenario is discussed by Gourgoulhon and Haensel [64]. Given specific assumptions about the equation of state of nuclear matter, they find that in the last stages of accretion that the matter will become less neutron-rich, and will emit a burst of $\bar{\nu}_{e}$ neutrinos with $\langle E\rangle \simeq 3 \mathrm{MeV}$. This lasts $\simeq 0.5 \mathrm{~ms}$ until truncated by black hole formation. At $10 \mathrm{kpc}$, we estimate that this would cause $\sim 3$ events above the SK threshold. In fact, since their model does not include neutrino opacities, the neutrino energy and the luminosity before the cutoff will both be lower. Thus, unless the neutron star is very close, this would be undetectable (see also Ref. [65] for a study of the sensitivity of LVD).

Thus there are some concrete models $[54,55,58]$ in which black hole formation occurs early enough to cut off the neutrino fluxes when they are still measurably high, though the uncertainties are large and depend on the details of the supernova models.

\section{Details of the neutrino signal}

In the general case, the observables for each neutrino flavor are the luminosity $L(t)$ and temperature $T(t)$ up to and during the time of black hole formation. The duration of the cutoff must be very short, of order the light crossing time $2 R / c \simeq 0.1 \mathrm{~ms}$. In the most detailed numerical treatment available [58], the duration of the cutoff is about $0.5 \mathrm{~ms}$. We assume that this will be typical for any mechanism of black hole formation. For black hole formation at very early times, the initial proto-neutron star would be larger than assumed in Ref. [58], and one might argue that this would lengthen the duration of the cutoff. However, it should be noted that what defines the cutoff is the increasing gravitational redshift, and this does not become large until the proto-neutron star is already very compact. For emission from the proto-neutron star, the neutrino gravitational redshifts are moderate; $z$ $\simeq G M / R c^{2} \sim 0.1$. The redshifts only become severe during the short cutoff at $t_{B H}$, when $z \rightarrow \infty$ (using the full expression for $z$ ). In any case, further modeling of the neutrino signal up to and during black hole formation is needed, especially for black hole formation at earlier times. It will be shown below that the statistical error in defining the position of the cutoff is larger than $0.5 \mathrm{~ms}$; therefore, all of the neutrino flavors can be considered to be cut off sharply and simultaneously at a time $t_{B H}$. These approximations can be made because the expected numbers of events during the cutoff are less than 1 .

In Ref. [58], some interesting details of the signal during the $\simeq 0.5 \mathrm{~ms}$ cutoff are pointed out. The very last neutrinos to be seen will not come from radial paths, but rather from unstable circular orbits. It should be noted that the calcula- 
tion of Ref. [58] only treats neutrinos on radial paths. The final decay of the luminosity due to the neutrinos on unstable circular orbits is expected to be exponential, with a time constant proportional to the black hole mass [66]. Since this time constant is very small, $\tau=3 \sqrt{3} G M_{B H} / c^{3} \simeq 0.04 \mathrm{~ms}$, the number of such events (proportional to the disregarded luminosity multiplied by this duration) will be negligible for the cases considered in this paper. Normally, electron neutrinos are emitted from the largest radius and with the lowest temperature. At the end of the neutrino signal during black hole formation, the electron neutrinos will be cut off last and will briefly have a higher temperature than the other flavors (due to less gravitational redshift). Unfortunately, all of these details of the transition are not observable with the present and proposed detectors, due to the limited statistics. For a very close supernova, the situation might be different; this will be discussed below.

The abrupt and simultaneous termination of all flavors of neutrinos allows a very simple mass test. Since the electron neutrino is nearly massless, the termination of the $\bar{\nu}_{e}$ event rate in SK will signal the black hole formation time $t_{B H}$ (the effects of a possible electron neutrino mass will be discussed below). Then, any events observed after $t_{B H}$ could only have come from neutral-current detection of time-of-flight delayed, massive $\nu_{\mu}, \nu_{\tau}, \bar{\nu}_{\mu}$, and $\bar{\nu}_{\tau}$. We have assumed that the detector background is negligible, in the sense that the expected number of background events over a typical delay time is $\ll 1$.

Before $t_{B H}$, one would like to measure $L(t)$ and $T(t)$ for all of the neutrino flavors. This is straightforward for $\nu_{e}$ and $\bar{\nu}_{e}$, since the detected outgoing lepton carries nearly the full neutrino energy in reactions with nuclear targets. Since $\nu_{\mu}$, $\nu_{\tau}, \bar{\nu}_{\mu}$, and $\bar{\nu}_{\tau}$ only have enough energy to undergo neutralcurrent interactions, they are indistinguishable. However, for the same reason, they are also expected to be produced with the same luminosity and temperature. It is not generally possible to measure the temperature for these species directly, and it must be inferred by the yields on different targets (cross sections with different energy dependence sample the spectrum differently; see Fig. 3 of Ref. [14]). The measurements of $L(t)$ and $T(t)$ for the various flavors before $t_{B H}$, as well as the value of $t_{B H}$ itself, are important probes of the supernova mechanism and the equation of state $[15,16]$. They will also be important for measuring the quantities needed for the mass measurement, in order to reduce the model dependence.

In the bulk of this paper, we concentrate in the analysis on mu and tau neutrino masses near the limit of detectability. The mass effects will then not appreciably affect the time dependence of the event rate except at the sharp cutoff at $t_{B H}$. In fact, it will be shown that only the luminosity and temperature at $t_{B H}$ itself are relevant. In the models $[54,55,58]$ considered, the neutrino luminosities and temperatures before $t_{B H}$ are roughly constant over the time scales of relevant mass delays. Thus it is adequate (and much more convenient analytically) to consider that the luminosities and temperatures of all flavors are constant for some period before the cutoff, and that they have simultaneous step-function cutoffs at $t_{B H}$. These assumptions will be relaxed below.

We assume the following temperatures: $T=3.5 \mathrm{MeV}$ for $\nu_{e}, T=5 \mathrm{MeV}$ for $\bar{\nu}_{e}$, and $T=8 \mathrm{MeV}$ for $\nu_{\mu}, \nu_{\tau}, \bar{\nu}_{\mu}$, and $\bar{\nu}_{\tau}$ [17]. This hierarchy is a consequence of the different opacities in the proto-neutron star, and the decreasing temperature with increasing radius. The temperatures in Ref. [58] were somewhat higher than these conventional values, but the authors explain that this is probably due to a numerical approximation in the transport code.

To illustrate our results quantitatively, we present results for two concrete cases. In the first, called "Early," black hole formation is assumed to occur a few $(\sim 1)$ seconds after core collapse, when the neutrino luminosities are of order $10^{52} \mathrm{erg} / \mathrm{s}$ per flavor. This case is nominally associated with black hole formation by accretion onto the proto-neutron star $[54,55]$. In the second, called 'Late," black hole formation is assumed to occur within several $(\simeq 10)$ seconds after core collapse, when the neutrino luminosities are of order $10^{51} \mathrm{erg} / \mathrm{s}$ per flavor. ${ }^{3}$ This case is nominally associated with black hole formation by a softening of the high-density equation of state in the proto-neutron star [58]. Direct extraction of the $\bar{\nu}_{e}$ luminosity from the SN 1987A data roughly supports the luminosity-time correspondences given here. It should be remembered that these are just examples-it will be shown that all of the necessary quantities can be measured in a realistic situation.

\section{NEUTRINO MASS EFFECTS}

\section{A. Detected event rate}

For a constant, normalized, thermal spectrum $f(E)$, but a general luminosity $L(t)$, the event rate for neutrinos with nonzero mass is

$$
\frac{d N}{d t}=\frac{N_{T}}{4 \pi D^{2}} \frac{1}{\langle E\rangle} \int_{0}^{\infty} d E f(E) \sigma(E) L(t-\Delta t(E))
$$

where $N_{T}$ is the number of targets in the detector, $D$ the supernova distance, and $\langle E\rangle$ the average energy (for a FermiDirac spectrum $\langle E\rangle=3.15 T)$. Generalization to a timedependent spectrum or a shape more general than FermiDirac would be straightforward. The argument of $L(t)$ is shifted to account for the possible energy-dependent delay of a massive neutrino.

As discussed above, we assume that the luminosity and temperature are constant before $t_{B H}$ (for at least much longer than the typical delay time), and then vanish abruptly. That is, $L(t)=L_{B H} \theta\left(t_{B H}-t\right)$, where $L_{B H}$ is the luminosity at the cutoff. In Eq. (2), we need to evaluate this with the delayed argument, i.e., $L(t-\Delta t(E))=L_{B H} \theta\left(t_{B H}-t+\Delta t(E)\right)$. For $t$ $<t_{B H}$, the step function is satisfied for all energies, and the event rate is

\footnotetext{
${ }^{3}$ Recent work of Pons et al. [16] suggests that black hole formation would occur after a few tens of seconds; however, their final luminosities are comparable to what we assume.
} 


$$
\frac{d N}{d t}(t)=C\left[\frac{L_{B H}}{10^{51} \mathrm{erg} / \mathrm{s}}\right] \int_{0}^{\infty} d E f(E)\left[\frac{\sigma(E)}{10^{-42} \mathrm{~cm}^{2}}\right] .
$$

The integral is the thermally-averaged cross section

$$
\sigma_{e f f}=\int_{0}^{\infty} d E f(E) \sigma(E) .
$$

This constant event rate is the same for massless neutrinos; as long as the delays are much less than the total duration of the supernova signal and the luminosity is constant, then at a given time the number lost by delays to later times is compensated by the number gained by delays from earlier times. This is not true at the start of the neutrino signal, but the rise is much less sharp than the black hole cutoff, is modeldependent, and is not considered further. For $t>t_{B H}$, there is an upper limit on the neutrino energy, which must be small enough for the neutrino to be delayed that long after $t_{B H}$. Then

$$
\frac{d N}{d t}(t)=C\left[\frac{L_{B H}}{10^{51} \mathrm{erg} / \mathrm{s}}\right] \int_{0}^{E_{\max }} d E f(E)\left[\frac{\sigma(E)}{10^{-42} \mathrm{~cm}^{2}}\right] .
$$

The upper limit $E_{\max }$ is simply the energy that makes the argument of the step function $\theta\left(t_{B H}-t+\Delta t(E)\right)$ vanish; using Eq. (1), this is

$$
E_{\max }=m \sqrt{\frac{0.515 D}{t-t_{B H}}}
$$

where the units are as in Eq. (1). Note that the neutrino mass and time dependence appear only through the limit of integration. If the neutrino energy can be measured, as in some charged-current reactions, then the event rates for separate ranges of neutrino energy can easily be obtained. For an $\mathrm{H}_{2} \mathrm{O}$ detector, the constant $C$ is

$$
C_{\mathrm{H}_{2} \mathrm{O}}=(1.74 / \mathrm{s})\left[\frac{M_{D}}{1 \mathrm{kton}}\right]\left[\frac{10 \mathrm{kpc}}{D}\right]^{2}\left[\frac{1 \mathrm{MeV}}{\langle E\rangle}\right] .
$$

The constant for a ${ }^{208} \mathrm{~Pb}$ detector can be obtained by scaling by the relative number of targets/kton, i.e., 18/208; therefore

$$
C_{208_{\mathrm{Pb}}}=(0.151 / \mathrm{s})\left[\frac{M_{D}}{1 \mathrm{kton}}\right]\left[\frac{10 \mathrm{kpc}}{D}\right]^{2}\left[\frac{1 \mathrm{MeV}}{\langle E\rangle}\right] .
$$

\section{B. Number delayed past $t_{B H}$}

The expected number of delayed counts $N_{d e l}$ after $t_{B H}$ can be determined analytically by integration of Eq. (5), which will be useful when $t_{B H}$ can be measured independently. This is simply

$$
\begin{aligned}
N_{d e l}= & \int_{t_{B H}}^{\infty} d t \frac{d N}{d t}(t) \\
= & C\left[\frac{L_{B H}}{10^{51} \mathrm{erg} / \mathrm{s}}\right] \int_{t_{B H}}^{\infty} d t \int_{0}^{\infty} d E \\
& \times f(E)\left[\frac{\sigma(E)}{10^{-42} \mathrm{~cm}^{2}}\right] \theta\left(t_{B H}-t+\Delta t(E)\right) \\
= & C\left[\frac{L_{B H}}{10^{51} \mathrm{erg} / \mathrm{s}}\right] \int_{0}^{\infty} d E f(E)\left[\frac{\sigma(E)}{10^{-42} \mathrm{~cm}^{2}}\right] \Delta t(E) .
\end{aligned}
$$

Note that the upper limit on energy in Eq. (5) was written using the step function $\theta\left(t_{B H}-t+\Delta t(E)\right)$; this step function then disappeared in the integration over $t$. Now define

$$
\langle\Delta t(E)\rangle_{f \sigma}=\frac{\int_{0}^{\infty} d E f(E) \sigma(E) \Delta t(E)}{\int_{0}^{\infty} d E f(E) \sigma(E)},
$$

where the $f \sigma$ subscript emphasizes that the weighting is over $f(E) \sigma(E)$, and not $f(E)$ alone (as for $\langle E\rangle$ ). Then

$$
N_{d e l}=\langle\Delta t(E)\rangle_{f \sigma} C\left[\frac{L_{B H}}{10^{51} \mathrm{erg} / \mathrm{s}}\right] \int_{0}^{\infty} d E f(E)\left[\frac{\sigma(E)}{10^{-42} \mathrm{~cm}^{2}}\right] \text {. }
$$

Recognizing the event rate before (or at) $t_{B H}$ from Eq. (3), this becomes

$$
N_{d e l}=\frac{d N}{d t}\left(t_{B H}\right) \times\langle\Delta t(E)\rangle_{f \sigma} .
$$

By use of Eq. (1), we see that Eq. (10) simply defines the average value of $1 / E^{2}$. By the mean-value theorem for integrals, this can be written as $1 / E_{c}^{2}$, where $E_{c}$ is a constant to be determined. The weighted delay can then be expressed as

$$
\langle\Delta t(E)\rangle_{f \sigma}=0.515\left(\frac{m}{E_{c}}\right)^{2} D .
$$

The physical significance of the "central" energy $E_{c}$ is that it is (to an excellent approximation) simply the Gamow peak of the falling thermal spectrum and the rising cross section. It can also be determined by numerical evaluation of Eq. (10). Thus we arrive at the very simple and important result:

$$
N_{d e l}=\frac{d N}{d t}\left(t_{B H}\right) \times 0.515\left(\frac{m}{E_{c}}\right)^{2} D,
$$

where the event rate is in $\mathrm{s}^{-1}$, and the other units are as in Eq. (1). This formula would obviously be true if only a single energy contributed and the sharp cutoff in the event rate (see Fig. 2 and Fig. 3 below) were simply rigidly translated by the delay. But it is remarkable and very convenient 
that it is still true even when there is a spectrum of energies and the event rate develops a decaying tail past the cutoff. As derived, this is an exact result.

In the derivation of these results we assumed that the luminosity and temperature (and hence also the event rate) were nearly constant before $t_{B H}$, as suggested by the results of Refs. $[54,55,58]$. For an arbitrary event rate, a fit can always be made to the event rate before $t_{B H}$, and $d N / d t$ at $t_{B H}$ extracted and used in the formula for $N_{d e l}$. (Below, we also discuss how $T$ and hence $E_{c}$ can be extracted from the data.) To integrate $N_{d e l}$ as above, it is only necessary that the event rate be approximately constant over the scale of the small possible mass delays, which is a very mild assumption.

Once the other quantities can be measured, then the neutrino mass $m$ is given by Eq. (14). We show how this can be done below.

\section{MEASUREMENT OF THE ELECTRON NEUTRINO MASS}

\section{A. CC event rate and measurement of $t_{B H}$}

We first consider how well $t_{B H}$ could be measured if we knew that $m_{\nu_{e}} \simeq 0$. The dominant event rate in SK is from $\bar{\nu}_{e}+p \rightarrow e^{+}+n$. The cross section [67] as a function of the neutrino energy $E$, including the recoil, weak magnetism, and radiative corrections, is well-approximated at typical supernova neutrino energies (where we can disregard the electron mass) by

$$
\sigma(E)=0.0952(E-1.3)^{2}(1-7 E / M),
$$

for neutrino energies $E>1.8 \mathrm{MeV}$. In this formula, $M$ is the nucleon mass in $\mathrm{MeV}$, and the cross section is in $10^{-42} \mathrm{~cm}^{2}$. For a temperature $T=5 \mathrm{MeV}$, the thermally-averaged cross section (for the sum of the two protons in $\mathrm{H}_{2} \mathrm{O}$ ) is $44 \times 10^{-42} \mathrm{~cm}^{2}$. (This is slightly smaller than the result used in Refs. $[12,68]$, due to an improved treatment of the corrections [67]). Thus, for a supernova at $10 \mathrm{kpc}$ as seen in SK (32 kton), the event rate due to $\bar{\nu}_{e}+p \rightarrow e^{+}+n$ can be easily calculated. Using Eq. (3), the rate just before the cutoff at $t_{B H}$ is $\simeq 1500 \mathrm{~s}^{-1}$ in the Early case and $\simeq 150 \mathrm{~s}^{-1}$ in the Late case. After $t_{B H}$, the rates are zero. We have disregarded the $0.5 \mathrm{~ms}$ duration [58] of the cutoff, which should contain about 0.4 events in the Early case and about 0.04 events in the Late case. Since these are fewer than 1, the cutoff can be considered to be sharp.

How is the cutoff time measured, and what is its error? Suppose we have an event rate $R(t)$ measured before the unknown cutoff time $t_{B H}$. The time of the last event $t_{\text {last }}$ is a lower bound for $t_{B H}$. If $t_{B H}$ were larger than $t_{\text {last }}$ by $\delta t$, then the number of events expected after $t_{\text {last }}$ would be $\delta N$ $\simeq R\left(t_{\text {last }}\right) \delta t$. If Poisson fluctuations caused that number $\delta N$ to fluctuate to 0 , then $t_{\text {last }}$ would be smaller than $t_{B H}$ by about $\delta t$. This can only occur for $\delta N \lesssim 1$, or $\delta t$ $\leqslant 1 / R\left(t_{\text {last }}\right)$. Thus, the error in determining the position of a sharp cutoff is generically of the form $1 / R\left(t_{\text {last }}\right)$, i.e., depending on the number of events $N$ as $1 / N$. For a rate with a tail instead of a sharp cutoff, the error in determining the offset time scales instead as $1 / \sqrt{N}$; see Ref. [18] for a dis- cussion of the differences. Furthermore, since $t_{\text {last }}$ is always less than $t_{B H}$, a bias correction $\simeq 1 / R\left(t_{\text {last }}\right)$ should be added to $t_{\text {last }}$ to estimate $t_{B H}$. A more sophisticated treatment of this problem using order statistics $[69,70]$ yields the same scaling results.

Thus, for the Early and Late cases, we find that $t_{B H}$ will be measured from the charged-current event rate in SK with precision slightly better than $\simeq 1 \mathrm{~ms}$ and $\simeq 10 \mathrm{~ms}$, respectively. These uncertainties on $t_{B H}$ will have a negligible effect on the mu and tau neutrino mass tests in the lead detector discussed below.

\section{B. Effects of a nonzero electron neutrino mass}

From the tritium beta decay experiments [5], the maximum allowed value of $m_{\nu_{e}}$ (by CPT, the same as $m_{\bar{\nu}_{e}}$ ) is about $3 \mathrm{eV}$. Using Eq. (5), it is straightforward to calculate the effects of $m_{v_{e}}=3 \mathrm{eV}$ on the $\bar{\nu}_{e}+p \rightarrow e^{+}+n$ event rate after $t_{B H}$ in SK. Suppose that $t_{B H}$ were somehow known independently. By calculating the event rate and integrating, in the Early case we find 21 events after the true $t_{B H}$, with delays as large as about $40 \mathrm{~ms}$. If unrecognized, this would bias the extracted $t_{B H}$ to be too large, and would seriously degrade the mu and tau neutrino mass test (looking ahead to Fig. 2). In the Late case, on the other hand, we would have only 2.1 events after the true $t_{B H}$, with delays as large as about $20 \mathrm{~ms}$, with less effect on the mu and tau neutrino mass test (see Fig. 3).

However, this potential problem in defining $t_{B H}$ due to the unknown electron neutrino mass can easily be avoided. In the reaction $\bar{\nu}_{e}+p \rightarrow e^{+}+n$ in a Cerenkov detector like SK, it is possible to measure the neutrino energy by measuring the positron energy and angle. At these energies,

$$
E_{\nu} \simeq\left(E_{e}+1.3\right)\left[1+\frac{E_{e}}{M}(1-\cos \theta)\right]
$$

where $E_{e}$ is the positron total energy in $\mathrm{MeV}, M$ is the nucleon mass, and $\cos \theta$ is for the positron along the neutrino direction. This follows from the two-body kinematics and the small neutron recoil; the full expression is given in Ref. [67]. From Eq. (1), different neutrino energies correspond to different delays. At a given time after $t_{B H}$, only energies low enough to have caused a delay that large are allowed. The maximum allowed energy, Eq. (6), falls very quickly after $t_{B H}$, as $1 / \sqrt{t-t_{B H}}$. Thus, different ranges of neutrino energy will be terminated at different times after $t_{B H}$, and these can be separated experimentally.

In general, one would use the event rate as a function of time and energy, Eq. (3) and Eq. (5), to make an unbinned maximum-likelihood fit to the measured neutrino energies and times to simultaneously measure both $m_{\nu_{e}}$ and $t_{B H}$. However, even without doing that, we can still get a good idea of how well we can measure $m_{\nu_{e}}$ and $t_{B H}$ by splitting the $\bar{\nu}_{e}+p \rightarrow e^{+}+n$ data into different ranges of neutrino energy, which we define as

Low: $0 \leqslant E \leqslant 11.3 \mathrm{MeV}$, 


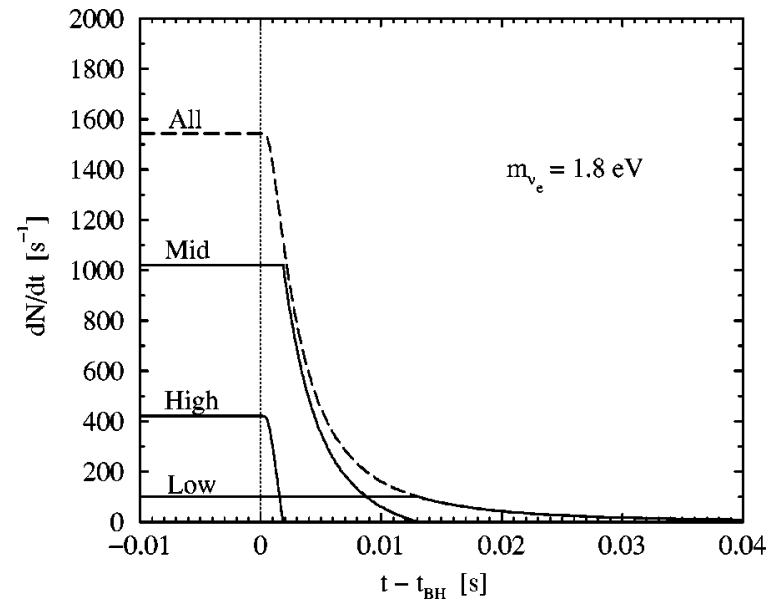

FIG. 1. The event rate due to $\bar{\nu}_{e}+p \rightarrow e^{+}+n$ in SK, in the Early case, with an assumed distance of $10 \mathrm{kpc}$. Note that only the rate after about $t_{B H}$ is shown, and that the range of $t-t_{B H}$ is very short. We took $m_{\nu_{e}}=1.8 \mathrm{eV}$, which is close to the minimum mass that can be discerned from this data. The labels "Low" (contains 2.4 events past the true $\left.t_{B H}\right)$, "Mid" (4.8 events), "High" (0.5 events), and "All" (7.7 events) refer to ranges of neutrino energy defined in the text.

$$
\begin{aligned}
& \text { Mid: } \quad 11.3 \leqslant E \leqslant 30 \mathrm{MeV}, \\
& \text { High: } \quad 30 \leqslant E \leqslant \infty \mathrm{MeV} .
\end{aligned}
$$

The Low group must be excluded from consideration because these events have positron total energy less than 10 $\mathrm{MeV}$, and can be confused with the 5-10 MeV gammas from the neutral-current reaction $\nu+{ }^{16} \mathrm{O} \rightarrow \nu+\gamma+X$, where $X$ is either $n+{ }^{15} \mathrm{O}$ or $p+{ }^{15} \mathrm{~N}$ [68]. In that energy range, one would not be able to distinguish delay effects due to $m_{\nu_{e}}$ or $m_{\nu_{\mu}}$ and $m_{\nu_{\tau}}$. Generally speaking, $m_{\nu_{e}}$ and $t_{B H}$ are correlated when extracted from the data (since $m_{\nu_{e}}>0$ has the effect of apparently increasing $t_{B H}$ ). However, the High group has much less delay and will thus primarily be sensitive to $t_{B H}$. Then the Mid group will principally be sensitive to $m_{\nu_{e}}$, by counting events delayed past the $t_{B H}$ determined by the High group.

In Fig. 1, we show such a possible analysis for the case of $m_{\nu_{e}}=1.8 \mathrm{eV}$, in the Early case. The numbers of events after the true $t_{B H}$ are: 2.4 (Low), 4.8 (Mid), and 0.5 (High). Since in the High group, the number of events in the tail is $\leqslant 1$, the cutoff appears sharp and the time of the last event (after the bias correction) specifies $t_{B H}$ to within the reciprocal of the event rate at the cutoff, i.e., about $2 \mathrm{~ms}$. This uncertainty affects the expected number in the Mid group by at most \pm 2 events. Even in this case, one can still reliably see a few delayed counts after the measured $t_{B H}$, enough to establish a nonzero mass (the statistics are discussed in detail in Sec. V). We have ignored the 0.4 events expected during the $0.5 \mathrm{~ms}$ duration of the cutoff.

Thus, in the Early case it will be realistically possible to probe electron neutrino masses as small as about $1.8 \mathrm{eV}$ in SK. The error on the time $t_{B H}$ extracted from the same data is not as large as the possible delays ( $\simeq 10 \mathrm{~ms}$, see Fig. 1), but instead depends on the statistics of the High data. Though from the High data alone the error on $t_{B H}$ is about 2 $\mathrm{ms}$, we anticipate that a more sophisticated fit to all of the data will reduce the error somewhat, to about $1 \mathrm{~ms}$. The smallest detectable $m_{\nu_{e}}$ could probably also be improved slightly.

In the Late case, the laboratory bound of $3 \mathrm{eV}$ on the electron neutrino mass will generally be stronger than that derived from the charged-current signal, and $t_{B H}$ will be measured to about $10 \mathrm{~ms}$.

\section{MEASUREMENT OF THE MU AND TAU NEUTRINO MASSES}

\section{A. General framework}

The basic signature of a mu or tau neutrino mass is the observation of neutral-current events after $t_{B H}$. If many counts delayed past $t_{B H}$ were observed, then Eq. (5) could be used to make an unbinned maximum likelihood fit to the mass based on how the rate fell off with time. The only measurable quantities for any delayed counts are their arrival times and their total number, since it is not possible to measure the neutrino energy in neutral-current interactions. This is simply because not enough kinematic variables are measured (the outgoing neutrino and the recoiling nucleus are not detected). In neutrino-electron scattering, measurement of the electron energy and angle would allow reconstruction of the neutrino energy in principle; in practice, the kinematic range of the outgoing electron angle is less than the angular resolution of the detectors. Thus it is not possible to select ranges of neutrino energy as in the $m_{\nu_{e}}$ measurement. While that could be done crudely by exploiting the different response functions of different targets (see Fig. 3 of Ref. [14]), it is not necessary if $t_{B H}$ is measured independently in SK. The various neutral-current yields can also be used to estimate the $\nu_{\mu}, \nu_{\tau}, \bar{\nu}_{\mu}$, and $\bar{\nu}_{\tau}$ temperature $T$ (or, more generally, the spectral shape).

The test proposed in this paper is to simply count the number of neutral-current events after $t_{B H}$. There is a very simple relation between the number of delayed counts and the mass, which we quote again because of its importance:

$$
N_{d e l}=\frac{d N}{d t}\left(t_{B H}\right) \times 0.515\left(\frac{m}{E_{c}}\right)^{2} D,
$$

where the event rate is in $\mathrm{s}^{-1}$, and the other units are as in Eq. (1). The first important point is that while there is a spectrum of energies, only one integral over that spectrum is important, i.e., the one that determines $E_{c}$. If instead we were making a maximum likelihood fit to a large number of delayed counts, the precise way the tail was filled out would depend on more details of the shape of $f(E) \sigma(E)$. The second important point is that after consideration of both the supernova neutrino model and the detector properties, the only remaining unknown is the neutrino mass. The cutoff time $t_{B H}$ can be measured in SK. The number of neutralcurrent counts $N_{d e l}$ will be measured between $t_{B H}$ and some 
TABLE I. This table shows how a given measured number of events $N$ determines a range for the allowed expected number of events $\mu$, using Poisson statistics. For the first line, $\mu=2.3$ is the largest expectation that yields $N=0$ at least $10 \%$ of the time. For the second line, $\mu=0.1$ is the smallest expectation that yields $N$ $=1$ (or greater) at least $10 \%$ of the time, and $\mu=3.9$ is the largest expectation that yields $N=1$ (or smaller) at least $10 \%$ of the time. Successive lines are similar. The best-fit $\mu$ is shown in parentheses. Using Eq. (18), which relates the number of events and the neutrino mass $m$, the corresponding allowed range in $m$ can be determined. Figures 4 and 5 can be used for the same purpose.

\begin{tabular}{lc}
\hline \hline Measured number & Allowed range of the expected number \\
\hline$N=0$ & $0.0 \leqslant \mu(\simeq 0.0) \leqslant 2.3$ \\
$N=1$ & $0.1 \leqslant \mu(\simeq 1.0) \leqslant 3.9$ \\
$N=2$ & $0.5 \leqslant \mu(\simeq 2.0) \leqslant 5.3$ \\
$N=3$ & $1.1 \leqslant \mu(\simeq 3.0) \leqslant 6.7$ \\
$N=4$ & $1.7 \leqslant \mu(\simeq 4.0) \leqslant 8.0$ \\
$N=5$ & $2.4 \leqslant \mu(\simeq 5.0) \leqslant 9.3$ \\
\hline \hline
\end{tabular}

suitable stopping point that depends on the size of the possible delay effects and the detector background rate. The neutral-current event rate at $t_{B H}$ due to mu and tau neutrinos will be measured with small error since it can be measured over an adequately long interval before $t_{B H}$. As noted, the central energy $E_{c}$ is well-approximated by the Gamow peak of the falling spectrum and the rising cross section. Thus $E_{c}$ depends on the temperature $T$; if not assumed from theory, this can be estimated from the data, as noted above. We assume that the distance $D$ can be determined by consideration of the total yield of events or by astronomical techniques (although a supernova at more than several kpc will be optically obscured by dust, it will still be visible at other wavelengths).

For given measured quantities, the best-fit mass is

$$
m=E_{c} \sqrt{\frac{N_{d e l}}{0.515 D \frac{d N}{d t}\left(t_{B H}\right)}} .
$$

In the likely case of no delayed events observed, then the best-fit mass is obviously $m \simeq 0$. A limit can be placed on the mass by considering the largest mass $m_{\text {lim }}$ that could have faked the massless case. At a chosen confidence level, this depends on the largest number of events that could have fluctuated down to 0 events. For example, using Poisson statistics, an expectation of 2.3 delayed counts fluctuates to 0 less than $10 \%$ of the time. Then $m_{\text {lim }}$ is obtained with Eq. (18) with $N_{d e l}$ set equal to 2.3. If $N_{d e l}>0$ is measured, Table I can be used to deduce the allowed range of the expected number of counts and hence the neutrino mass. Since the fractional error on $N_{d e l}$ due to Poisson statistics is large $(\simeq 1 / \sqrt{2.3} \simeq 65 \%)$, errors on other inputs are expected to be irrelevant. If a large number of counts were measured, the Poisson relative error would be smaller, and the uncertainties on the inputs would play a more important role.
Dropping all constants of proportionality, we can also write $m_{l i m}$ as

$$
m_{\text {lim }} \sim E_{c} \sqrt{\frac{\langle E\rangle D}{\sigma_{e f f} L_{B H} M_{D}}} .
$$

While no longer written in terms of the directly measured quantities, this has the advantage of showing the dependence on the theoretical inputs more explicitly. For a supernova that does not have the sharp cutoff in the rate characteristic of black hole formation, the model-independent $\langle t\rangle$ analysis $[12,13]$ yields an $m_{\text {lim }}$ that is independent of the distance $D$ and that scales with the detector mass $M_{D}$ as $1 / M_{D}^{1 / 4}$ [13]. The different scaling with $D$, and the much more favorable scaling with $M_{D}$, are consequences of the sharp cutoff in the neutrino flux in the present case.

\section{B. Supernova neutrino detection in lead}

Recently, there has been discussion of building a large supernova detector based on ${ }^{208} \mathrm{~Pb}$ [71-74]. A lead detector would observe supernova neutrinos by detecting neutrons produced through both neutral-current and charged-current interactions of the neutrinos with the lead nuclei. The neutrons would be produced primarily by the neutral-current interactions of $\nu_{\mu}, \nu_{\tau}, \bar{\nu}_{\mu}$, and $\bar{\nu}_{\tau}$, because these have the highest temperature. The neutrons could be detected in (for example) a liquid scintillator doped with $\simeq 0.1 \%$ gadolinium, which has a very large neutron-capture cross section, yielding an $8 \mathrm{MeV}$ gamma cascade. The neutron capture time in such a doped scintillator is very short, of order 0.030 ms [75], much smaller than the typical mass delays.

A novel scheme based on a clear solution of lead perchlorate is also being explored [76]. Neutrons would be detected by the $8.6 \mathrm{MeV}$ gamma cascade from capture on ${ }^{35} \mathrm{Cl}$, and electrons would be detected by their Cerenkov light.

The neutral-current cross sections for neutrinos and antineutrinos on ${ }^{208} \mathrm{~Pb}$ have been calculated by Hargrove et al. [71] and Fuller et al. [77]. The calculations in this paper are based on the Fuller et al. cross section. ${ }^{4}$ While the Hargrove et al. and the Fuller et al. results for the spectrum-averaged cross sections agree within $20 \%$ at $T=8 \mathrm{MeV}$, the underlying calculations are quite different. As discussed, the cross section uncertainties have only a minor effect on the mass test if $N_{d e l}$ is small. Nevertheless, a laboratory measurement of the neutrino cross sections on lead (perhaps with the ORLAND detector [79] at the Spallation Neutron Source) would be valuable.

Hargrove et al. consider only the allowed contribution. The cross section is assumed to be dominated by a narrow M1 resonance at $8 \mathrm{MeV}$, so that

$$
\sigma(E) \sim(E-8 \mathrm{MeV})^{2},
$$

\footnotetext{
${ }^{4}$ A very recent calculation by Kolbe and Langanke [78] suggests a lower neutral-current cross section for neutrinos on ${ }^{208} \mathrm{~Pb}$, although the differences with the standard Fuller, Haxton, and McLaughlin [77] cross section remain unexplained.
} 


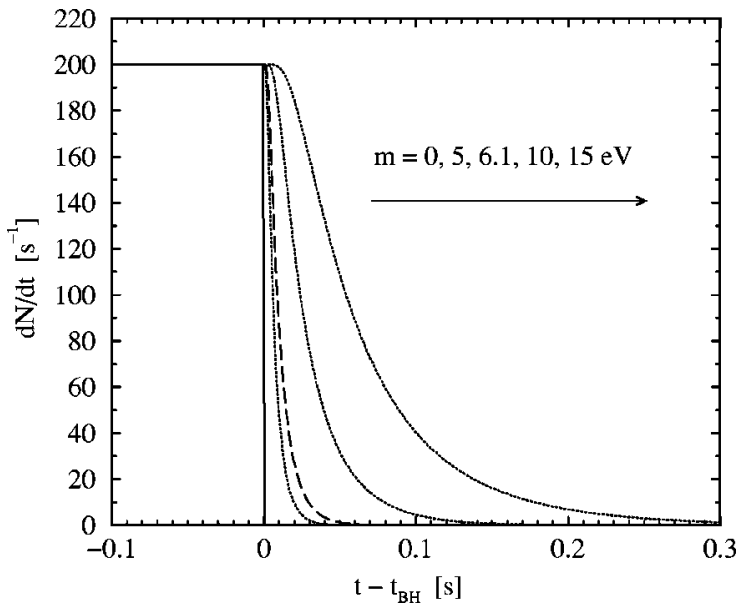

FIG. 2. The results for the combined 1-n neutral-current event rate due to $\nu_{\mu}, \nu_{\tau}, \bar{\nu}_{\mu}$, and $\bar{\nu}_{\tau}$ in OMNIS. Note that only the rate after about $t_{B H}$ is shown. The Early case is assumed, with $t_{B H}$ occurring a few $(\sim 1)$ seconds after core collapse, and luminosities of $10^{52} \mathrm{erg} / \mathrm{s}$ per flavor at $t_{B H}$. The assumed distance is $10 \mathrm{kpc}$. Before $t_{B H}$, there are other reactions that produce neutrons; they are not included here, and those events will have to be statistically subtracted from the measured neutron rate. Maximal $\nu_{\mu} \leftrightarrow \nu_{\tau}$ mixing with small $\delta m^{2}$ is assumed, so $m \simeq m_{\nu_{2}} \simeq m_{\nu_{3}}$. The $m=0$ case is drawn with a solid line. The $m=6.1 \mathrm{eV}$ case, with 2.3 events expected in the tail, is the first case that can be reliably distinguishable from $m=0$, and is drawn with a long-dashed line. The results for other masses are drawn with dotted lines.

for neutrino energies $E>8 \mathrm{MeV}$. However, Fuller et al. find that the cross section is dominated by the first-forbidden contribution (they also point out some apparent errors in the Hargrove et al. calculation of the allowed contribution). Fuller et al. do not provide the cross sections as a function of neutrino energy, but instead only provide thermally-averaged results for various assumed spectra. However, it is straightforward to make a reasonable fit to $\sigma(E)$ itself. The neutralcurrent cross section is dominated by excitations to the giant dipole resonance at $80 \mathrm{MeV} / A^{1 / 3} \simeq 14 \mathrm{MeV}$. This is just below the 2-neutron emission threshold, and they find the 2 -neutron emission probability to be very low ( $\leqslant 5 \%$ of all neutrons). The cross section can be fit by the form

$$
\sigma(E) \sim(E-14 \mathrm{MeV})^{2},
$$

for neutrino energies $E>14 \mathrm{MeV}$. A fit was made to the Fuller et al. results, summing the allowed and forbidden (for $T=8 \mathrm{MeV}$, the latter is about $80 \%$ of the total) contributions, and summing the results for $\nu$ and $\bar{\nu}$ (for either $\nu_{\mu}$ or $\nu_{\tau}$ channel). Using this form, the leading constant was found to be $2.7 \times 10^{-42} \mathrm{~cm}^{2}$. After fitting, the thermally-averaged cross sections in the first six columns of Table I of Ref. [77] were matched to better than $10 \%$. The 1-neutron spallation probability is approximately independent of energy over the relevant range, and can be taken to be 0.90 . It should be emphasized that our fits to the cross section and branching ratio will only be valid over the limited range of energy that

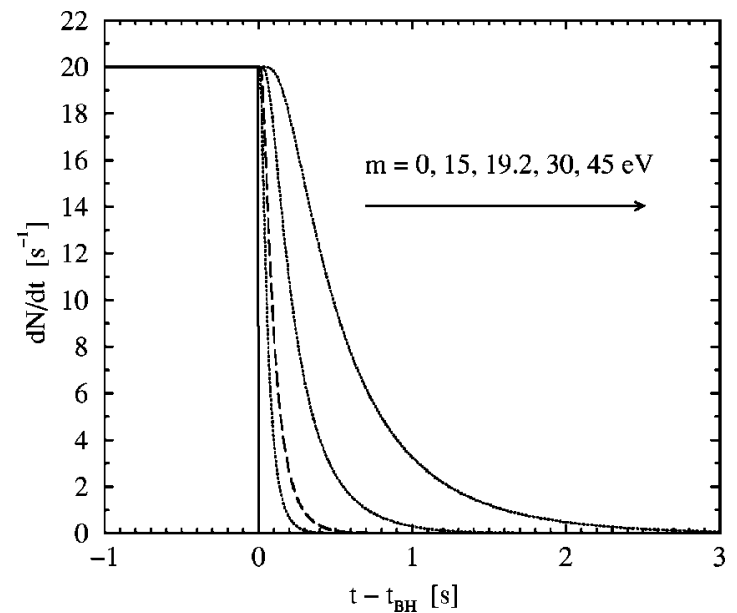

FIG. 3. As in Fig. 2, except that the Late case is assumed, with $t_{B H}$ occurring within several $(\simeq 10)$ seconds after core collapse, and luminosities of $10^{51} \mathrm{erg} / \mathrm{s}$ per flavor at $t_{B H}$. The $m=19.2 \mathrm{eV}$ case, with 2.3 events expected in the tail, is the first case that can be reliably distinguishable from $m=0$, and is drawn with a longdashed line. Note the changes of scale on the axes.

we consider. For a temperature $T=8 \mathrm{MeV}$, the thermallyaveraged cross section in Eq. (4) for the sum of $\nu$ and $\bar{\nu}$ (again, for either $\nu_{\mu}$ or $\nu_{\tau}$ ) on ${ }^{208} \mathrm{~Pb}$, including the 1-neutron spallation probability, is about $760 \times 10^{-42} \mathrm{~cm}^{2}$. For a supernova at $10 \mathrm{kpc}$ in which the neutrino fluxes are not terminated by black hole formation, the number of 1-neutron neutral-current events due to $\nu_{\mu}, \nu_{\tau}, \bar{\nu}_{\mu}$, and $\bar{\nu}_{\tau}$, all at $T$ $=8 \mathrm{MeV}$, is 455 events in 1 kton of ${ }^{208} \mathrm{~Pb}$ with perfect neutron detection efficiency, in agreement with Ref. [77] (who use $T=7.9 \mathrm{MeV}$ ).

It should be noted that the calculations above were specifically for ${ }^{208} \mathrm{~Pb}$, which is $52 \%$ of the abundance of natural lead. On the basis of the Thomas-Reiche-Kuhn sum rule, Fuller et al. [77] argue that the total neutral-current neutrino cross sections at these energies should scale as $\sigma \sim A$, where $A$ is the mass number. Thus, the total cross sections for the three isotopes of lead should be very similar. The position of the giant dipole resonance changes only as $\sim 1 / A^{1 / 3}$, and the 2-neutron emission thresholds are $0.7 \mathrm{MeV}$ higher in ${ }^{206} \mathrm{~Pb}$ and ${ }^{207} \mathrm{~Pb}$; therefore, 1-neutron emission will also dominate in these isotopes.

\section{Results for a lead detector}

In this section, we calculate results for a ${ }^{208} \mathrm{~Pb}$ detector that is specified by the number of events expected for a supernova at $10 \mathrm{kpc}$ in which the neutrino fluxes are not cut off by black hole formation. We assume that the detector will have $\simeq 10001$-neutron neutral-current events due to $\nu_{\mu}, \nu_{\tau}$, $\bar{\nu}_{\mu}$, and $\bar{\nu}_{\tau}$ in this case. A possible design for a 4-kton lead detector with about this many events is described by Boyd [73]. This design also includes $10 \mathrm{kton}$ of iron, with a smaller number of neutral-current events (not included in our calculations). Further refinements in the cross section and detector design $[73,74]$ (and hence the neutron detection efficiency) may affect the mass of lead required to meet the 


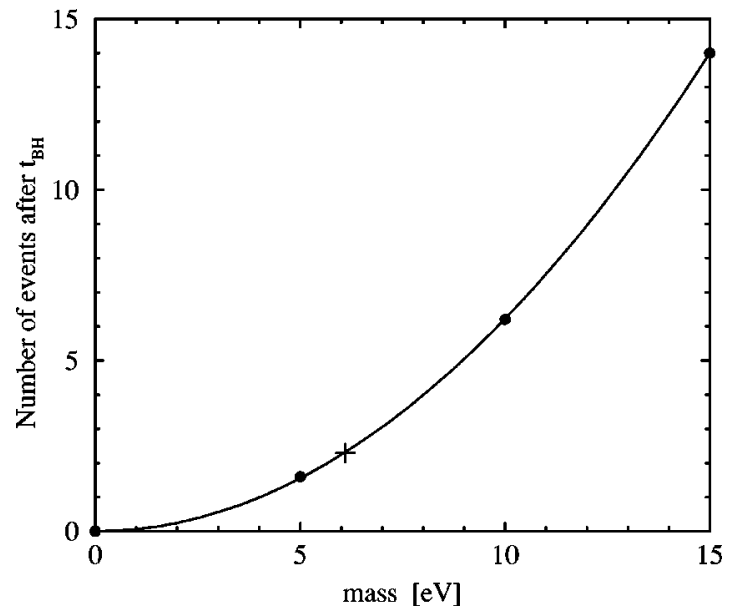

FIG. 4. The expected number of delayed counts $N_{d e l}$ (those after $t_{B H}$, due to the mass effects) in OMNIS as a function of the neutrino mass. The calculation uses the same assumptions as in Fig. 2, the Early case. The points are obtained by direct numerical integration. The "+" indicates the smallest discernible mass at the $90 \%$ C.L. The solid line is obtained with Eq. (17), using $E_{c}$ $=40.7 \mathrm{MeV}$, the Gamow peak energy.

design goal of $\simeq 1000$ neutral-current events of this type. Using the Fuller et al. [77] cross section, this goal could be met with a 2.2 kton lead detector with perfect neutron detection efficiency. We refer to this lead detector, whatever its eventual precise specifications, as the OMNIS (Observatory for Multiflavor NeutrInos from Supernovae) detector.

In the following, we assume a supernova distance of 10 kpc. Using the product of the thermally-averaged cross section and the branching ratio given above, the event rates due to neutral-current detection of $\nu_{\mu}, \nu_{\tau}, \bar{\nu}_{\mu}$, and $\bar{\nu}_{\tau}$ can easily be calculated with Eq. (3) and Eq. (5). These rates are shown in Fig. 2 and Fig. 3 for the Early and Late cases. Recall that the luminosities and cutoff times chosen are simply examples; in a real case, the relevant quantities will be measured, not assumed. In particular, $t_{B H}$ will be measured using the $\bar{\nu}_{e}+p \rightarrow e^{+}+n$ events in SK.

In Fig. 4 for the Early case and in Fig. 5 for the Late case, the number of delayed events $N_{d e l}$ (that is, $\nu_{\mu}, \nu_{\tau}, \bar{\nu}_{\mu}$, and $\bar{\nu}_{\tau}$ events after $\left.t_{B H}\right)$ is shown versus the neutrino mass. The points are from direct numerical integration of Eq. (5), and the solid line is the simple analytic result of Eq. (17). Note that $E_{c}=40.7 \mathrm{MeV}$ is calculated using the Gamow peak of $f(E) \sigma(E)$, and is not fitted.

In order to use Eq. (18), a minor correction to the measured event rate before $t_{B H}$ must be made. In a lead detector, one expects to measure just the total neutron rate. Thus the expected contributions from the charged-current 1-neutron and 2-neutron events will have to be statistically subtracted, along with the contributions of $\nu_{e}$ and $\bar{\nu}_{e}$ to the neutralcurrent rate. The subtracted rate of neutrons before $t_{B H}$ is about $20 \%$ of the total [77].

The cross section normalization appears only in the event rate, where it is multiplied by $L_{B H}$, which is a priori unknown. Only their product, in the form of the measured event rate, is needed in Eq. (18). The cross section shape

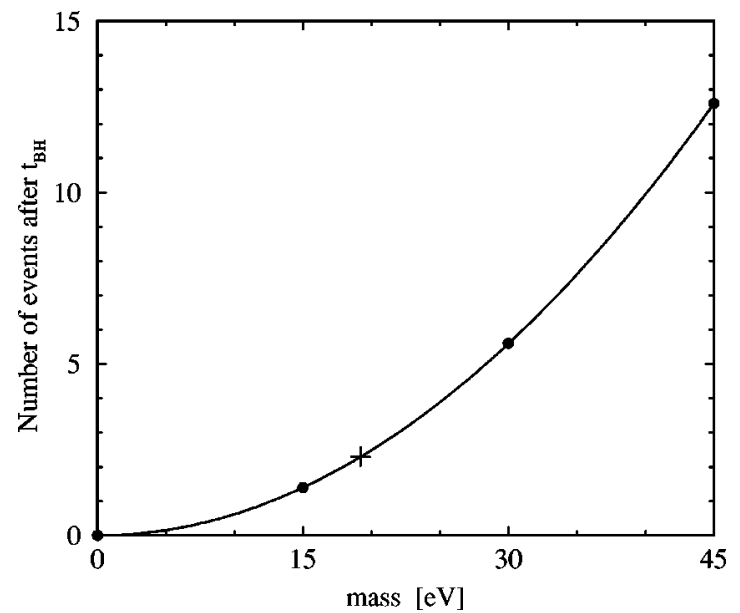

FIG. 5. As in Fig. 4, but for the Late case, and with the assumptions of Fig. 3. Note the change in the horizontal scale.

only affects $E_{c}$. Using a Fermi-Dirac spectrum with temperature $T=8 \mathrm{MeV}$, then $E_{c} \simeq 41 \mathrm{MeV}$ using the Fuller et al. [77] cross section given above, and $E_{c} \simeq 35 \mathrm{MeV}$ using the Hargrove et al. [71] cross section given above; this is a negligible difference. The spectral temperature $T$ (nominally $8 \mathrm{MeV}$ ) of the mu and tau neutrinos at the time of the cutoff is a priori unknown, perhaps by $\pm 25 \%$, and this also affects $E_{c}$. The heavy-flavor temperature can be estimated from the data by the yields on different targets (see Fig. 3 of Ref. [14]), and this may reduce the uncertainty on $T$. Thus, in terms of impact on the measurement of the neutrino mass, the uncertainties in the thermally-averaged neutral-current cross section on ${ }^{208} \mathrm{~Pb}$ are of less importance than the Poisson counting error.

Using Figs. 4 and 5, we obtain mass sensitivity as low as $6.1 \mathrm{eV}$ in the Early case and $19.2 \mathrm{eV}$ in the Late case. These are the first masses that can be reliably discerned (90\% C.L.) from the massless case, since they correspond to at least 2.3 expected events after $t_{B H}$. Larger masses give even more delayed events, and hence are easier to measure. In these results, we have assumed that $\nu_{\mu}$ and $\nu_{\tau}$ are maximally mixed, with $\delta m^{2} \simeq 10^{-3} \mathrm{eV}^{2}$, as suggested by the atmospheric neutrino results [10], so that both contribute to $N_{d e l}$. The results for the neutrino mass will then apply to the two relevant mass eigenstates. If we do not consider this mixing, then perhaps only the tau (or mu) neutrinos will have a mass and be delayed. Then $N_{d e l}$ is half as large as assumed here, and by Eq. (18), $m_{\text {lim }}$ is $\sqrt{2}$ larger. Since assuming that only one neutrino is massive is the most conservative possibility, the deduced limit would in fact apply for either of the mu and tau neutrino masses.

Finally, we discuss some sources of error for the number of delayed events $N_{d e l}$ in a ${ }^{208} \mathrm{~Pb}$ detector, all of which are negligible. We ignore possible detector backgrounds over the short time scale of possible delays. The duration of the cutoff is about $0.5 \mathrm{~ms}$ [58]; taking that into account would make $N_{\text {del }}$ larger by $\simeq 0.5 \times 200 \times 0.0005=0.05$ events in the Early case and 0.005 events in the Late case. As noted, the uncertainty on $t_{B H}$ from SK is assumed to be about $1 \mathrm{~ms}$ in the Early case and $10 \mathrm{~ms}$ in the Late case. From Fig. 2 and Fig. 
3 , this uncertainty can be seen to change the expected number $N_{d e l}$ by $\simeq \pm 200 \times 0.001= \pm 0.2$ events in the Early case and $\simeq \pm 20 \times 0.010= \pm 0.2$ events in the Late case. Even with $m_{\nu_{e}} \lesssim 1.8 \mathrm{eV}$ determined in SK, there can still be some $\nu_{e}$ and $\bar{\nu}_{e}$ events (charged- and neutral-current on ${ }^{208} \mathrm{~Pb}$ ) after the true $t_{B H}$. In the worst case, assuming no tagging on 2-neutron events or events with an electron, the $\nu_{e}$ and $\bar{\nu}_{e}$ events contribute about $20 \%$ of the total neutron rate before $t_{B H}$. Assuming $m_{\nu_{e}}=1.8 \mathrm{eV}$ and $E_{c} \simeq 30 \mathrm{MeV}$, then the number of these events after the true $t_{B H}$ is $\simeq 50 \times 0.515$ $\times(1.8 / 30)^{2}=0.09$ in the Early case and 0.009 in the Late case. For a larger lead detector or a closer supernova, some of these errors could become relevant.

\section{Results for SNO}

The principal neutral-current reactions available in SNO are $\nu+d \rightarrow \nu+p+n$ and $\bar{\nu}+d \rightarrow \bar{\nu}+p+n$, detected by neutron capture. For a supernova at $10 \mathrm{kpc}$ in which the neutrino fluxes are not truncated by black hole formation, 485 events are expected, of which 400 would be caused by $\nu_{\mu}, \nu_{\tau}, \bar{\nu}_{\mu}$, and $\bar{\nu}_{\tau}$ [13]. Perfect neutron detection efficiency is assumed. Before $t_{B H}$, the neutral-current event rate due to these flavors may be obtained by scaling the ${ }^{208} \mathrm{~Pb}$ results by $400 /$ 1000 , the ratio of the total numbers of events expected for a supernova that does not form a black hole. This works simply because both the event rate before $t_{B H}$ and the total number of events have the same dependence on $\sigma_{\text {eff }}$ and the number of targets. Then, using Eq. (18) with $E_{c}=32 \mathrm{MeV}$ [13] and $N_{d e l}=2.3$, we obtain $m_{l i m}=8 \mathrm{eV}$ in the Early case and $m_{\text {lim }}=24 \mathrm{eV}$ in the Late case.

However, it may not be possible to reach this sensitivity in practice due to the long neutron capture time in heavy water (an exponential distribution with time constant $\tau_{n}$ ). The value of $\tau_{n}$ depends on the neutron capture technique: with the dissolved $\mathrm{MgCl}_{2}$ salt, $\tau_{n} \simeq 4 \mathrm{~ms}$; with the ${ }^{3} \mathrm{He}$ counters, $\tau_{n} \simeq 16 \mathrm{~ms}$; and with pure $\mathrm{D}_{2} \mathrm{O}, \tau_{n} \simeq 35 \mathrm{~ms}$ [80]. The effect of this smearing is to delay events after $t_{B H}$ even in the massless case:

$$
N_{d e l} \rightarrow N_{d e l}+\frac{d N}{d t}\left(t_{B H}\right) \times \tau_{n} .
$$

For the Early case, this adds $0.8\left(\tau_{n} / 10 \mathrm{~ms}\right)$ events after $t_{B H}$. Thus, unless the salt is used, the neutrino mass sensitivity of SNO will be degraded because events after $t_{B H}$ can be delayed by either $\nu_{\mu}$ and $\nu_{\tau}$ mass effects or the nonzero neutron capture time.

\section{E. Results for SK}

The first set of neutral-current reactions available in SK are those on ${ }^{16} \mathrm{O}$ discussed above that yield a 5-10 MeV gamma in the final state [68]. For $\nu_{\mu}, \nu_{\tau}, \bar{\nu}_{\mu}$, and $\bar{\nu}_{\tau}, 710$ events in total are expected for a supernova at $10 \mathrm{kpc}$ [12]. Before $t_{B H}$, the neutral-current event rate may be obtained by scaling the ${ }^{208} \mathrm{~Pb}$ results by $710 / 1000$. In practice, this event rate will be obtained from the measured one by statistically subtracting the comparable rate due to low-energy $\bar{\nu}_{e}+p \rightarrow e^{+}+n$ events, which are indistinguishable in SK. Using Eq. (18) with $E_{c}=60 \mathrm{MeV}$ [12] and $N_{d e l}=2.3$, we obtain $m_{\text {lim }}=11 \mathrm{eV}$ in the Early case and $m_{\text {lim }}=34 \mathrm{eV}$ in the Late case.

However, it may not be possible to reach this sensitivity in practice because of the low-energy $\bar{\nu}_{e}+p \rightarrow e^{+}+n$ events after $t_{B H}$, of which there can be as many as 2.4 in the Early case, due to the limited sensitivity to $m_{\nu_{e}}$ in SK. Furthermore, the very steep cross section on ${ }^{16} \mathrm{O}$ is much more sensitive to the temperature or the spectral shape in general (see Fig. 3 of Ref. [14]), and so this result is more modeldependent. Thus the mu and tau neutrino mass sensitivity of SK using the neutral-current reactions on ${ }^{16} \mathrm{O}$ will be limited.

The second set of neutral-current reactions available in SK are $\nu+e^{-} \rightarrow \nu+e^{-}$and $\bar{\nu}+e^{-} \rightarrow \bar{\nu}+e^{-}$, for which 120 events due to $\nu_{\mu}, \nu_{\tau}, \bar{\nu}_{\mu}$, and $\bar{\nu}_{\tau}$ are expected for a supernova at $10 \mathrm{kpc}$ [12]. Before $t_{B H}$, the event rate for these reactions may be obtained by scaling the ${ }^{208} \mathrm{~Pb}$ results by $120 / 1000$. One must first subtract from the measured event rate events due to $\nu_{e}+e^{-} \rightarrow \nu_{e}+e^{-}, \bar{\nu}_{e}+e^{-} \rightarrow \bar{\nu}_{e}+e^{-}$, and $\bar{\nu}_{e}+p \rightarrow e^{+}+n$ in the forward cone. The unwanted events dominate the signal before $t_{B H}$ by a factor of $\simeq 5$, so the statistical subtraction will introduce some error. If this effect can be ignored, then using Eq. (18) with $E_{c}=25 \mathrm{MeV}$ [12] and $N_{d e l}=2.3$, we obtain $m_{\text {lim }}=11 \mathrm{eV}$ in the Early case and $m_{\text {lim }}=34 \mathrm{eV}$ in the Late case.

However, it may not be possible to reach this sensitivity in practice, again because of the limited sensitivity to $m_{v_{e}}$, which can allow otherwise indistinguishable $\nu_{e}$ and $\bar{\nu}_{e}$ events after $t_{B H}$. In the Early case, we estimate that there could be $\simeq 0.9$ such events after $t_{B H}$. Thus, the mu and tau neutrino mass sensitivity of SK using the neutral-current reactions on electrons will also be limited.

\section{DISCUSSION AND CONCLUSIONS}

\section{A. Distance dependence of the neutrino mass sensitivity}

Throughout this paper, we have assumed that the next Galactic supernova will be at a distance of $10 \mathrm{kpc}$. In the Bahcall-Soneira Galactic model [23,24], 25\%, 50\%, and $75 \%$ of supernovae are within about 7,10 , and $14 \mathrm{kpc}$ of Earth, respectively. If the events during the short $(\simeq 0.5 \mathrm{~ms})$ cutoff can be disregarded, then the results for other distances can be scaled with Eq. (19), and are shown in Fig. 6 and Fig. 7. Other errors, for example the error in $N_{d e l}$ that comes from the small error on $t_{B H}$, are independent of $D$ in their relative importance.

A close supernova at $1 \mathrm{kpc}$ would obviously have 100 times as many events as we have assumed, and would naively have mass sensitivity about 3 times better than at 10 $\mathrm{kpc}$, i.e., about $2 \mathrm{eV}$ in the Early case. However, there could be a number of events during the short cutoff that would make defining $t_{B H}$ more difficult than for a more distant supernova (even assuming that the high event rate in SK does not saturate the detector). Assuming a $\simeq 0.5 \mathrm{~ms}$ duration [58], there could be 40 such events in SK in the Early case and about 4 in the Late case. Note that these are estimated simply by the area of the triangle with height given by 


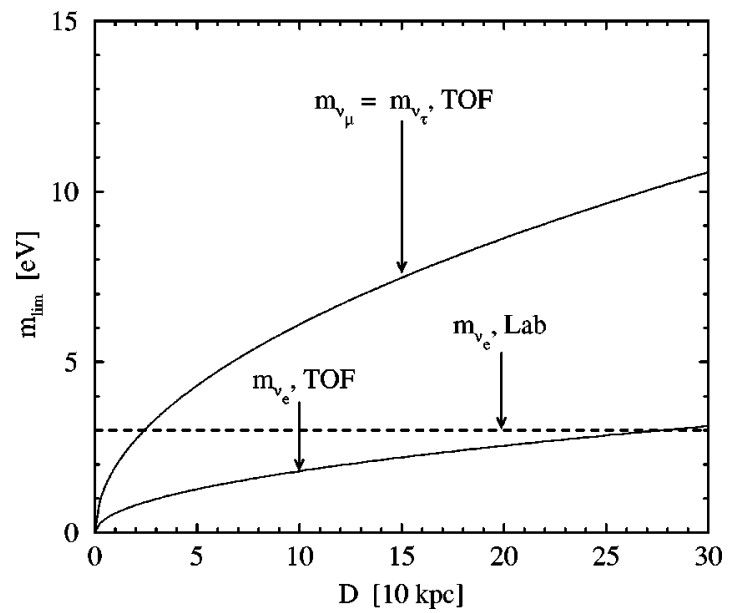

FIG. 6. The mass sensitivity as a function of the supernova distance (solid lines), for the Early case, for $m_{\nu_{\mu}} \simeq m_{\nu_{\tau}}$ measured in the OMNIS detector, and for $m_{\nu_{e}}$ measured in SK. This figure is appropriate if $N_{d e l}=0$ is measured and only a limit is being placed on the neutrino mass (if $N_{d e l}>0$ is measured and hence a nonzero mass is discovered, see Table I). The dashed line is the present laboratory upper limit on $m_{\nu_{e}}$ [5]. In using Eq. (19) to make this figure, we assumed that the events in the $\simeq 0.5 \mathrm{~ms}$ tail can be disregarded. Depending on the unknown details of the tail, this assumption will break down at perhaps $\sim 3 \mathrm{kpc}$ and the mass sensitivity will not improve further with decreasing distance.

the event rate at $t_{B H}$ and width given by $0.5 \mathrm{~ms}$. In fact, the neutrino temperatures are falling rapidly during these $0.5 \mathrm{~ms}$, due to increasing gravitational redshift; taking that and the detection threshold into account would reduce these numbers. Even if $t_{B H}$ could be defined with negligible error, there could still be neutral-current events after $t_{B H}$ due to the $\simeq 0.5 \mathrm{~ms}$ duration of the cutoff: perhaps 5 events in OMNIS in the Early case and 0.5 events in the Late case. Again, these are conservatively large estimates. The presence of events during the cutoff would weaken the mass sensitivity, and it would no longer decrease with decreasing distance. However, the real behavior of the luminosity and temperature during the cutoff is not well known, and further modeling along the lines of Baumgarte et al. [58] is needed.

For an extremely close (and hence rare) supernova, e.g., Betelgeuse at $\sim 0.1 \mathrm{kpc}$, the possibilities are even greater, particularly for exploring the process of black hole formation $[58,59]$, provided that the neutrino observatories can accommodate the enormous event rates.

\section{B. Neutrino oscillations}

While a full discussion of neutrino oscillations is beyond the scope of this paper, we make a few brief comments. Oscillations of $\nu_{\mu} \leftrightarrow \nu_{\tau}$ are not important in the sense that these flavors cannot be distinguished experimentally. The atmospheric neutrino results suggest that both are massive, with a small mass difference and a large mixing angle [10]; if so, the measured mass corresponds to the nearly degenerate mass eigenstates. Oscillations of $\nu_{\mu}, \nu_{\tau} \rightarrow \nu_{s}$ will decrease the number of neutral-current events; this is irrelevant

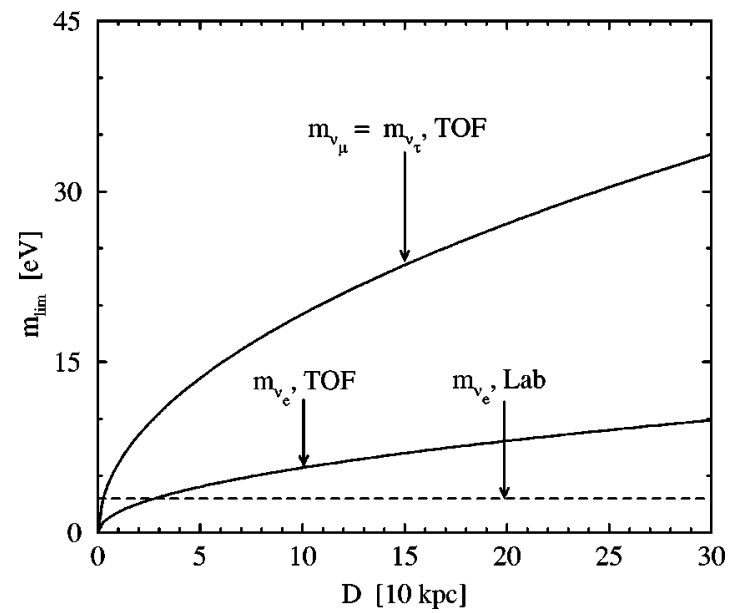

FIG. 7. As in Fig. 6, but for the Late case. Because of the lower luminosity, the mass sensitivity may flatten out only below about $\sim 1 \mathrm{kpc}$. Note the change in the vertical scale.

in the sense that the mass measurement depends on the measured, not predicted, event rate at $t_{B H}$. Oscillations of $\nu_{\mu}, \nu_{\tau} \leftrightarrow \nu_{e}$ (and their antiparticles) can in principle complicate the mass tests. However, because of the higher temperature for $\nu_{\mu}$ and $\nu_{\tau}$, such oscillations would greatly increase the number of charged-current events and would harden the electron or positron spectrum; see, e.g., Refs. [77,81]. If evidence of such oscillations were seen, the formalism presented here could easily be enlarged to include oscillations. The positron spectrum from $\bar{\nu}_{e}+p \rightarrow e^{+}+n$ from SN1987A appears to exclude large $\bar{\nu}_{\mu}, \bar{\nu}_{\tau} \leftrightarrow \bar{\nu}_{e}$ mixing [82].

\section{Conclusions}

If a black hole forms early in a core-collapse supernova, then the fluxes of the various flavors of neutrinos will be abruptly and simultaneously terminated when the neutrinospheres are enveloped by the event horizon. For a massive neutrino, the cutoff in the arrival time will be delayed by $\Delta t \sim(m / E)^{2}$ relative to a massless neutrino. The SK detector can measure both $t_{B H}$ and $m_{\nu_{e}}$ by the arrival times of lowand high-energy $\bar{\nu}_{e}+p \rightarrow e^{+}+n$ events, for which the neutrino energies can be measured. The mu and tau neutrinos are detectable only by their neutral-current interactions, in which their energies are not measured. However, their masses can be measured by counting the number of these neutral-current events detected after $t_{B H}$.

The mass sensitivity depends on the supernova neutrino luminosity $L_{B H}$ at cutoff, the distance $D$, and the detector used. For luminosities of $10^{52} \mathrm{erg} / \mathrm{s}$ per flavor at cutoff (the Early case), and a distance of $10 \mathrm{kpc}$, SK will be able to measure an electron neutrino mass as small as $1.8 \mathrm{eV}$ and OMNIS would be able to measure $m_{\nu_{\mu}} \simeq m_{\nu_{\tau}}$ as small as about $6 \mathrm{eV}$. These results are perhaps even slightly conservative, as the luminosities in Refs. [54,55] were in fact a few times larger than assumed in the Early case. As discussed, the mu and tau neutrino masses were assumed to be degenerate because of the atmospheric neutrino results [10]; in this 
case the masses are really those of the relevant mass eigenstates.

Using the neutral-current channels in SNO and SK, the neutrino mass sensitivity is nominally $\simeq 10 \mathrm{eV}$ for each. However, it appears that various practical effects will degrade those results.

For other luminosities, distances, and detector masses, the mass sensitivity scales as in Eq. (19), i.e.,

$$
m_{l i m} \sim \sqrt{\frac{D}{L_{B H} M_{D}}} .
$$

This should be contrasted with the case in which the neutrino luminosities are not truncated by black hole formation, where $m_{\text {lim }} \sim 1 / M_{D}^{1 / 4}$ and is independent of $D$.

As we have discussed, there seems to be a good chance that the ongoing and proposed neutrino detectors can observe the truncation of the neutrino signals caused by black hole formation in a Galactic core-collapse supernova. This would have profound consequences, even if no delayed events were observed and only limits were placed on the neutrino masses.
Besides the obvious astrophysical importance of such an observation, this could improve the limit on the tau neutrino mass by a factor of almost $10^{7}$. Moreover, the technique discussed in this paper is the only known possibility for direct measurement of the $\nu_{\mu}$ and $\nu_{\tau}$ masses (either Dirac or Majorana) in the crucial $\mathrm{eV}$ range suggested by the indirect neutrino mass tests $[1,2,4,8]$ discussed in the Introduction.

\section{ACKNOWLEDGMENTS}

J.F.B. was supported by Caltech for the initial portion of this work, and by the NASA/Fermilab Astrophysics Center (supported by the DOE and NASA under NAG5-7092) for the latter portion. R.N.B. was supported by NSF grant PHY9901241. A.M. was supported at the Oak Ridge National Laboratory, managed by UT-Battelle, LLC, for the U.S. Dept. of Energy under contract DE-AC05-00OR22725. We thank Felix Boehm, Steve Bruenn, Will Farr, Josh Grindlay, Manoj Kaplinghat, Gail McLaughlin, Alex Murphy, YongZhong Qian, Robert Shrock, Petr Vogel, and Jerry Wasserburg for discussions.
[1] S. S. Gershtein and Y. B. Zel'dovich, Zh. Éksp. Teor. Fiz., Pis'ma Red. 4, 174 (1966) [JETP Lett. 4, 120 (1966)]; R. Cowsik and J. McClelland, Phys. Rev. Lett. 29, 669 (1972); E. W. Kolb and M. S. Turner, The Early Universe (AddisonWesley, Menlo Park, CA, 1990).

[2] C. P. Ma, Nucl. Phys. B (Proc. Suppl.) 51, 254 (1996); W. Hu, D. J. Eisenstein, and M. Tegmark, Phys. Rev. Lett. 80, 5255 (1998); M. Kaplinghat, R. E. Lopez, S. Dodelson, and R. J. Scherrer, Phys. Rev. D 60, 123508 (1999); A. R. Cooray, Astron. Astrophys. 348, 31 (1999); R. A. Croft, W. Hu, and R. Dave, Phys. Rev. Lett. 83, 1092 (1999); M. Fukugita, G. Liu, and N. Sugiyama, ibid. 84, 1082 (2000); E. Gawiser, astro-ph/0005365.

[3] M. Kawasaki, K. Kohri, and N. Sugiyama, Phys. Rev. Lett. 82, 4168 (1999); Phys. Rev. D 62, 023506 (2000); G. F. Giudice, E. W. Kolb, and A. Riotto, ibid. (to be published), hep-ph/0005123; G. F. Giudice, E. W. Kolb, A. Riotto, D. V. Semikoz, and I. I. Tkachev, hep-ph/0012317.

[4] V. Barger, T. J. Weiler, and K. Whisnant, Phys. Lett. B 442, 255 (1998).

[5] Ch. Weinheimer et al., Phys. Lett. B 460, 219 (1999); V. M. Lobashev et al., ibid. 460, 227 (1999).

[6] K. Assamagan et al., Phys. Rev. D 53, 6065 (1996).

[7] R. Barate et al., Eur. Phys. J. C 2, 395 (1998).

[8] F. Vissani, J. High Energy Phys. 06, 022 (1999); M. Czakon, J. Studnik, and M. Zralek, hep-ph/0006339.

[9] L. Baudis et al., Phys. Rev. Lett. 83, 41 (1999).

[10] Y. Fukuda et al., Phys. Rev. Lett. 81, 1562 (1998); S. Fukuda et al., ibid. 85, 3999 (2000).

[11] R. E. Shrock, Phys. Lett. 96B, 159 (1980); Phys. Rev. D 24, 1232 (1981); 24, 1275 (1981).

[12] J. F. Beacom and P. Vogel, Phys. Rev. D 58, 053010 (1998).

[13] J. F. Beacom and P. Vogel, Phys. Rev. D 58, 093012 (1998).
[14] J. F. Beacom, in Neutrinos in the Next Millennium, edited by G. Domokos and S. Kovesi-Domokos (World Scientific, Singapore, 2000), hep-ph/9909231.

[15] H. T. Janka, astro-ph/9505034.

[16] J. A. Pons, S. Reddy, M. Prakash, J. M. Lattimer, and J. A. Miralles, Astrophys. J. 513, 780 (1999); J. A. Pons, J. A. Miralles, M. Prakash, and J. M. Lattimer, astro-ph/0008389.

[17] J. R. Wilson and R. W. Mayle, Phys. Rep. 227, 97 (1993); M. Herant, W. Benz, W. R. Hix, C. L. Fryer, and S. A. Colgate, Astrophys. J. 435, 339 (1994); A. Burrows, J. Hayes, and B. A. Fryxell, ibid. 450, 830 (1995); H.-T. Janka and E. Müller, Astron. Astrophys. 306, 167 (1996); A. Mezzacappa et al., Astrophys. J. 495, 911 (1998); C. L. Fryer and A. Heger, ibid. 541, 1033 (2000).

[18] J. F. Beacom and P. Vogel, Phys. Rev. D 60, 033007 (1999).

[19] J. F. Beacom, R. N. Boyd, and A. Mezzacappa, Phys. Rev. Lett. 85, 3568 (2000).

[20] S. van den Bergh, Comments. Astrophys. 17, 125 (1993); G. A. Tammann, W. Löffler, and A. Schröder, Astrophys. J., Suppl. Ser. 92, 487 (1994).

[21] S. van den Bergh, Phys. Rep. 204, 385 (1991).

[22] B. Aschenbach, Nature (London) 396, 141 (1998); A. F. Iyudin et al., ibid. 396, 142 (1998).

[23] J. N. Bahcall and R. M. Soneira, Astrophys. J. Lett. 238, L17 (1980).

[24] J. N. Bahcall and T. Piran, Astrophys. J. Lett. 267, L77 (1983).

[25] R. G. Strom, Astron. Astrophys. 288, L1 (1994).

[26] K. Hatano, A. Fisher, and D. Branch, Mon. Not. R. Astron. Soc. 290, 360 (1997).

[27] W. D. Arnett, D. N. Schramm, and J. W. Truran, Astrophys. J. Lett. 339, L25 (1989).

[28] S. van den Bergh, Astron. J. 99, 843 (1990).

[29] P. M. Dragicevich, D. G. Blair, and R. R. Burman, Mon. Not. 
R. Astron. Soc. 302, 693 (1999).

[30] M. L. Cherry et al., J. Phys. G 8, 879 (1982); G. T. Zatsepin and O. G. Ryazhskaya, Usp. Fiz. Nauk 146, 713 (1985) [Sov. Phys. Usp. 28, 726 (1985)]; D. Joutras et al., Astrophys. Lett. Commun. 27, 257 (1988); K. S. Hirata, ICRR-239-91-8, Ph.D. thesis, 1991; M. Aglietta et al., Astropart. Phys. 1, 1 (1992); E. N. Alexeyev et al., Zh. Éksp. Teor. Fiz. 104, 2897 (1993) [JETP 77, 339 (1993)]; R. S. Miller et al., Astrophys. J. 428, 629 (1994); M. Ambrosio et al., Astropart. Phys. 8, 123 (1998).

[31] A. Abramovici et al., Science 256, 325 (1992); B. C. Barish, gr-qc/9905026.

[32] C. Dupraz et al., Astron. Astrophys. 324, 683 (1997).

[33] F. X. Timmes, R. Diehl, and D. H. Hartmann, Astrophys. J. 479, 760 (1997).

[34] W. C. Haxton and C. W. Johnson, Nature (London) 333, 325 (1988).

[35] M. Prakash, S. Reddy, J. M. Lattimer, and P. J. Ellis, Heavy Ion Phys. 4, 271 (1996); J. M. Lattimer and M. Prakash, Phys. Rep. 333, 121 (2000); H. Heiselberg and V. Pandharipande, Annu. Rev. Nucl. Part. Sci. 50, 481 (2000).

[36] W. Zhang, T. E. Strohmayer, and J. H. Swank, Astrophys. J. Lett. 482, L167 (1997); N. K. Glendenning, S. Pei, and F. Weber, Phys. Rev. Lett. 79, 1603 (1997); M. C. Miller, F. K. Lamb, and D. Psaltis, Nucl. Phys. B (Proc. Suppl.) 69, 123 (1998); M. C. Miller and F. K. Lamb, Astrophys. J. Lett. 499, L37 (1998); B. Link, R. I. Epstein, and J. M. Lattimer, Phys. Rev. Lett. 83, 3362 (1999); H. Heiselberg and M. HjorthJensen, Astrophys. J. Lett. 525, L45 (1999); N. K. Glendenning and F. Weber, astro-ph/0003426.

[37] K. Hirata et al., Phys. Rev. Lett. 58, 1490 (1987); K. S. Hirata et al., Phys. Rev. D 38, 448 (1988).

[38] R. M. Bionta et al., Phys. Rev. Lett. 58, 1494 (1987); C. B. Bratton et al., Phys. Rev. D 37, 3361 (1988).

[39] L. Zampieri, M. Colpi, S. L. Shapiro, and I. Wasserman, Astrophys. J. 505, 876 (1998).

[40] G. E. Brown, S. W. Bruenn, and J. C. Wheeler, Comments. Astrophys. 16, 153 (1992); G. E. Brown and J. C. Weingartner, Astrophys. J. 436, 843 (1994).

[41] K. Ratnatunga and S. van den Bergh, Astrophys. J. 343, 713 (1989).

[42] C. L. Fryer, Astrophys. J. 522, 413 (1999).

[43] F. X. Timmes, S. E. Woosley, and T. A. Weaver, Astrophys. J. 457, 834 (1996).

[44] H. Umeda, K. Nomoto, and T. Nakamura, astro-ph/9912248.

[45] A. Akmal, V. R. Pandharipande, and D. G. Ravenhall, Phys. Rev. C 58, 1804 (1998).

[46] G. E. Brown and H. A. Bethe, Astrophys. J. 423, 659 (1998).

[47] H. A. Bethe and G. E. Brown, Astrophys. J. Lett. 445, L129 (1995).

[48] E. Ergma and E. P. J. van den Heuvel, Astron. Astrophys. 331, L29 (1998).

[49] E. P. J. van den Heuvel and G. M. H. J. Habets, Nature (London) 309, 598 (1984).

[50] S. F. P. Zwart, F. Verbunt, and E. Ergma, Astron. Astrophys. 321, 207 (1997).

[51] J. E. Grindlay, http://www.aas.org/publications/baas/v31n5/ aas195/1026.htm
[52] Y. Z. Qian, P. Vogel, and G. J. Wasserburg, Astrophys. J. 494, 285 (1998).

[53] C. Sneden et al., Astrophys. J. Lett. 533, L139 (2000).

[54] A. Burrows, Astrophys. J. 334, 891 (1988); see also 300, 488 (1986).

[55] A. Mezzacappa and S.W. Bruenn, in The Identification of Dark Matter, edited by N.J.C. Spooner and V. Kudryavtsev (World Scientific, Singapore, 1999).

[56] J. R. Wilson, Astrophys. J. 163, 209 (1971).

[57] J. I. Kapusta and K. A. Olive, Phys. Rev. Lett. 64, 13 (1990); N. K. Glendenning and S. A. Moszkowski, ibid. 67, 2414 (1991); V. Thorsson, M. Prakash, and J. M. Lattimer, Nucl. Phys. A572, 693 (1994).

[58] T. W. Baumgarte, S. A. Teukolsky, S. L. Shapiro, H. T. Janka, and W. Keil, Astrophys. J. 468, 823 (1996).

[59] W. Keil and H.-Th. Janka, Astron. Astrophys. 296, 145 (1995).

[60] N. K. Glendenning, Astrophys. J. 448, 797 (1995).

[61] T. W. Baumgarte, S. L. Shapiro, and S. A. Teukolsky, Astrophys. J. 458, 680 (1996).

[62] T. W. Baumgarte, S. L. Shapiro, and S. A. Teukolsky, Astrophys. J. 443, 717 (1996).

[63] S. A. Colgate, Astrophys. J. 163, 221 (1971); R. A. Chevalier, ibid. 346, 847 (1989).

[64] E. Gourgoulhon and P. Haensel, Astron. Astrophys. 271, 187 (1993).

[65] W. Fulgione and G. Navarra, Astron. Astrophys. 347, 119 (1999).

[66] W. L. Ames and K. S. Thorne, Astrophys. J. 151, 659 (1968); S. L. Shapiro, Phys. Rev. D 40, 1858 (1989).

[67] P. Vogel and J. F. Beacom, Phys. Rev. D 60, 053003 (1999).

[68] K. Langanke, P. Vogel, and E. Kolbe, Phys. Rev. Lett. 76, 2629 (1996).

[69] R. V. Hogg and A. T. Craig, Introduction to Mathematical Statistics, 4th ed. (Macmillan, New York, 1978).

[70] W. T. Eadie et al., Statistical Methods in Experimental Physics (American Elsevier, New York, 1971).

[71] C. K. Hargrove, I. Batkin, M. K. Sundaresan, and J. Dubeau, Astropart. Phys. 5, 183 (1996).

[72] P. F. Smith, Astropart. Phys. 8, 27 (1997).

[73] R. N. Boyd, in Origins of Matter and Evolution of Galaxies, edited by T. Kajino and S. Kubono (World Scientific, Singapore, 2000).

[74] J. J. Zach, A. S. Murphy, D. Marriott, and R. N. Boyd (unpublished).

[75] F. Boehm et al., Phys. Rev. D 62, 073002 (2000).

[76] P. J. Doe, S. R. Elliott, C. Paul, and R. G. Robertson, Nucl. Phys. B (Proc. Suppl.) 87, 512 (2000); S. R. Elliott, Phys. Rev. C 62, 065802 (2000).

[77] G. M. Fuller, W. C. Haxton, and G. C. McLaughlin, Phys. Rev. D 59, 085005 (1999).

[78] E. Kolbe and K. Langanke, Phys. Rev. C 63, 025802 (2001).

[79] F. T. Avignone et al., in Perspectives in Nuclear Physics, edited by J. H. Hamilton, H. K. Carter, and R. B. Piercey (World Scientific, Singapore, 1999).

[80] J. Boger et al., Nucl. Instrum. Methods Phys. Res. A 449, 172 (2000).

[81] T. K. Kuo and J. Pantaleone, Phys. Rev. D 37, 298 (1988); S. Choubey, D. Majumdar, and K. Kar, J. Phys. G 25, 1001 (1999); A. S. Dighe and A. Y. Smirnov, Phys. Rev. D 62, 
033007 (2000); G. Dutta, D. Indumathi, M. V. Murthy, and G. Rajasekaran, ibid. 61, 013009 (2000).

[82] A. Y. Smirnov, D. N. Spergel, and J. N. Bahcall, Phys. Rev. D 49, 1389 (1994); B. Jegerlehner, F. Neubig, and G. Raffelt, ibid. 54, 1194 (1996); see also more recent work: C. Lunardini and A. Y. Smirnov, ibid. (to be published), hep-ph/0009356; H. Minakata and H. Nunokawa, hep-ph/0010240; M. Kachelriess, R. Tomas, and J. W. Valle, hep-ph/0012134; K. Takahashi, M. Watanabe, and K. Sato, hep-ph/0012354. 